\title{
Quantum Control of Laser-driven Chiral Molecular Motors
}

\author{
Masahiro Yamaki ${ }^{1}$, Sheng H. Lin ${ }^{1,2}$, Kunihiko Hoki ${ }^{3}$ and Yuichi Fujimura ${ }^{3}$ \\ ${ }^{1}$ Department of Applied Chemistry, National Chiao Tung University, \\ Hsinchu, Taiwan 30010 \\ 2Institute of Atomic and Molecular Sciences, Academia Sinica, Taipei, Taiwan 10617 \\ ${ }^{3}$ Department of Chemistry, Graduate School of Science, Tohoku University, \\ Sendai, Japan 980-8578
}

\section{Introduction}

The design and control of functional molecular machines and devices is one of the fascinating and challenging research targets in molecular science (Feringa et al., 2000; Kinbara \& Aida, 2005; Kay et al., 2007). They were originally inspired from biological machines such as ATP synthases (Boyer, 1993; Abrahams et al., 1994) and myosin and kinesin (Jülicher et al., 1997). They now include various kinds of artificial molecular machines such as transmitters, shuttles, nanocars and logic gates (Balzani et al., 2008), which can be driven by external forces at the molecular level. Some of them are not simply sizeddown versions of macroscopic machines and are controlled at the quantum level (Roncaglia \& Tsironis, 1998).

Lasers are energy sources over a wide range of wave lengths from mid-infrared to ultraviolet, which make it possible to drive various sizes of molecular machines without any direct contact. Lasers are expected to play an important role as a source of external forces for controlling molecular machines because lasers have various controlling-parameters such as central frequencies, pulse shapes, photon polarizations and time differences between two pulses (Assion et al., 1998; Gouliemakis et al., 2004).

Based on coherent control theory (Kosloff et al. 1989; Shi \& Rabitz, 1990; Shapiro \& Brumer, 2000), laser pulses can be designed to produce the maximum desired target with minimum laser energy (Assion et al., 1998; Rice \& Zhao, 2000; Gordon \& Fujimura, 2002; Bandrauk et al., 2002). Molecular machines can be controlled through coherent interactions between lasers and molecules at a quantum level (Hoki et al., 2003). The procedures are sometimes called "quantum ignition" for driving molecular motors (Fujimura et al., 2004). The time evolution is obtained by solving the time-dependent Schrödinger equation or the Liouville equation (Sugawara \& Fujimura, 1994; Ohtsuki et al., 1999; Hoki et al., 2001). Application of coherent control theory enables extraction of key factors for driving molecular motors with a unidirectional motion, though we have to wait for further experimental progress to carry out coherent control experiments on artificial molecular machines. In this chapter, we present fundamental principles for unidirectional motions of chiral molecular motors driven by linearly polarized laser pulses having no photon helicity. 
In Sec. 2, we first clarify the role of molecular chirality. We discuss the mechanism of unidirectional motions of chiral motors. For this purpose, it is instructive to mention mechanisms of unidirectional motions of bio-motors. In bio-motors, the unidirectional motions are explained in terms of so-called nonequilibrium fluctuations of a Brownian motion with a saw-toothed ratchet potential (Astumian \& Hänggi, 2002; Reimann, 2002). Molecular chirality is an essential factor for the unidirectional motion of a rotary motor driven by a linearly polarized laser pulse. The motion is basically determined by both the asymmetric potential originating from its molecular chirality and a periodic perturbation of laser-molecule interactions (Hoki et al., 2003).

We next present the results of a quantum dynamics simulation of simple, real chiral molecules to clarify the mechanism of unidirectional motions. The directional motion is determined by molecular chirality, not by the external laser field, which gives periodic perturbations to motors. We treat molecular motors in a heat bath by using the Liouville equation to demonstrate dephasing effects due to interactions with the heat bath. Here, we consider the case in which the magnitude of fluctuation forces causing dephasing is weak compared with that of laser-motor interactions. The situation is common for the treatment of molecule-laser interactions but different from the case of bio-motors, the dynamics of which can be explained in terms of nonequilibrium fluctuations of bath modes. We also present results of unidirectional motions obtained by using a pump-dump laser-ignition method (Hoki et al., 2004). We finally present a practical method for detecting quantum dynamics of molecular motors in real time.

In Sec. 3, we briefly present results of optimal control for unidirectional motions of chiral molecular motors. A local and global control methods were applied to chiral molecular motors (Yamaki, 2005; Yamaki, 2008).

In Sec. 4, we treat a simple molecular machine consisting of two internal rotors, one of which is a propeller and the other of which is a motor driven by laser pulses. We discuss the mechanism of energy transmission from the motor to the propeller.

In Sec. 5, we present some future research subjects in laser-driven molecular motors after a summary of the chapter.

\section{Unidirectional rotations of molecular motors}

First of all, we define the equation of motion and the Hamiltonian of the light-driven molecular motors, which are used throughout this chapter. The system Hamiltonian of a molecular motor in the presence of an electric field of light $E(t)$ within the long-wave approximation is written as

$$
\hat{H}_{S}^{\Gamma}(t)=\hat{H}_{0}^{\Gamma}-\hat{\boldsymbol{\mu}}^{\Gamma} \cdot \boldsymbol{E}(t),
$$

where $\hat{H}_{0}^{\Gamma}$ is a molecular Hamiltonian that consists of a kinetic energy operator, $\hat{T}$ and a potential energy operator, $\hat{V}_{0}^{\Gamma} ; \hat{\mu}^{\Gamma}$ is a dipole moment vector; $\Gamma(=S, R)$ indicates molecular chirality, which is sometimes omitted if it is not necessary in the later sections. Explicit forms of $\hat{H}_{S}^{\Gamma}(t)$ will be defined later. The total Hamiltonian including surrounding effects is written as

$$
\hat{H}_{S}^{\Gamma}(t)=\hat{H}_{S}^{\Gamma}(t)+\hat{H}_{B}+\hat{H}_{S B}^{\Gamma}
$$


with the bath Hamiltonian $\hat{H}_{B}$ and the motor-bath interaction Hamiltonian $\hat{H}_{S B}^{\Gamma}$. We can obtain results of interactions between the motor and laser pulses as a special case. The quantum dynamics of the molecular motors can be generally described by the timedependent Liouville equation given as

$$
i \hbar \frac{\partial}{\partial t} \hat{\rho}^{\Gamma}(t)=\hat{L}^{\Gamma}(t) \hat{\rho}^{\Gamma}(t)
$$

where $\hat{\rho}^{\Gamma}(t)$ denotes the density operator of molecular motors in the heat bath, and $\hat{L}^{\Gamma}(t)=\left[\hat{H}^{\Gamma}(t)\right.$, ] with commutator $[$,$] . The time-dependent coupled equation (3) is solved$ numerically with an initial condition $\hat{\rho}^{\Gamma}(0)$ given by the Boltzmann distribution at temperature $T$.

\subsection{Molecular chirality and periodically perturbed chiral molecular motors}

One of the ideas for creating unidirectional motions is shown in the upper panel of Fig. 1. Consider a saw-toothed ratchet potential $V_{0}(\phi)$. The potential $V_{0}(\phi)$ is characterized by the $L$ periodicity as $V_{0}(\phi)=V_{0}(\phi+L)$, and by the broken spatial symmetry that is expressed as $V_{0}(\phi-c) \neq V_{0}(-\phi+c)$ for any $c$. The asymmetric static potential energy $V_{0}(\phi)$ is not sufficient to create unidirectional motion of the system, and one of the typical ratchet systems introduces a time-correlated tilting force $f(t)$, which is known as a nonequilibrium fluctuation of a Brownian motion. As shown in the upper-left panel of Fig. 1, when the force is negative, a mass point falls down to the left side. On the other hand, when the force is positive, the mass point falls down to the right side. In this way, by combining static asymmetric potential $V_{0}(\phi)$ and unbiased force such that the time average of $f(t)$ equals zero, a unidirectional motion in the system can be obtained. Here, the direction is determined by the shape of the static asymmetric potential energy $V_{0}(\phi)$, and the mass point moves in the intuitive direction in which the slope to climb is gentle.

A rotary motion of a chiral molecule can also be characterized by asymmetric potential energy. Consider an idealized chiral molecule with two rigid groups, $A$ and $B$, as shown in Fig. 2. Here, the mass of $A$ is set to be heavy compared with that of $B$, and $A$ is taken as the body and $\mathrm{B}$ is taken as the rotating group. These two groups are connected to each other by a single bond, and the rotary motion is described in terms of coordinate $\phi$. We call a pair of the chiral molecules $(S)$ - and $(R)$-motors in this paper. The potential energy of rotation $V^{\Gamma}(\phi)$ with $\Gamma=S$ or $R$ is characterized by an asymmetric potential, and the potential energy satisfies $V^{S}(\phi)=V^{R}(-\phi)$ since the two motors $(S)$ and $(R)$ have mirror image. The rigid group $\mathrm{B}$ has plus and minus extremities to create an electric dipole moment. Therefore, the lowest-order interaction energy between the dipole moment and an oscillating electric field with a linear photon polarization can be written as $\cos (\phi) f(t)$. We note that a linearly polarized light has no photon helicity, i.e., no photon angular momentum.

The time-dependent effective potential energy of the rotary motion under an oscillating electric field is schematically shown in the lower panel of Fig. 1. The major difference from the abovementioned tilting ratchet is that the chiral molecule is periodically perturbed, and the entire effective potential has $L$ periodicity as $V^{\Gamma}(\phi, t)=V^{\Gamma}(\phi+L, t)$. In contrast to the case of the tilting ratchet, it is not always obvious whether the system creates a unidirectional motion or not, although the same rotational direction as that in the upper panel in Fig. 1 is expected. In the next subsection, we examine the direction of the rotary motion by using a real model molecule. 


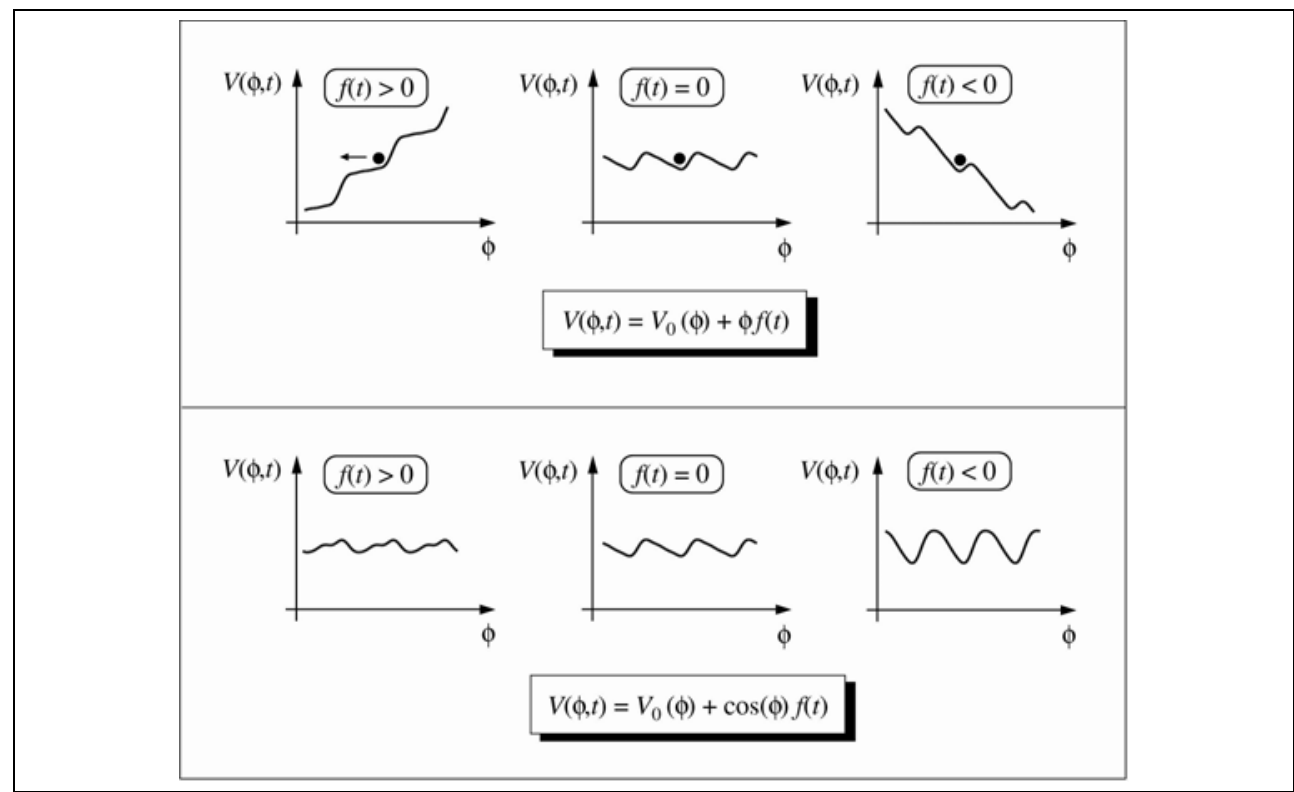

Fig. 1. Upper panel: a model for unidirectional Brownian motions. Lower panel: a model for unidirectional motions of a chiral molecular motor induced by a linearly polarized laser pulse.

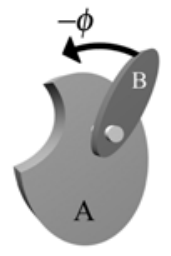

(S)-motor

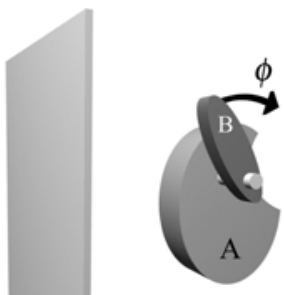

(R)-motor

Fig. 2. A simplified model for chiral molecular motors, $(S)$ - and $(R)$-motors, which are mirror images of each other. They have two rigid groups, A and B. The parameter $\phi$ denotes the rotational angle between $\mathrm{A}$ and $\mathrm{B}$.

\subsection{Quantum dynamics simulation of a chiral molecular motor}

We chose (R)-2-chloro-5-methylcyclopenta-2,4-dienecarbaldehyde shown in Fig. 3a as a model system. Note that the molecule has a chiral center at the $\mathrm{C}^{3}$ atom. Consider the internal rotation of the $\mathrm{CHO}$ group around the $\mathrm{C}^{2}-\mathrm{C}^{3}$ bond, and the coordinate of the rotation $\phi$ as the dihedral angle $\mathrm{O}^{1}-\mathrm{C}^{2}-\mathrm{C}^{3}-\mathrm{H}^{4}$. In the molecular frame, the $z$-axis is chosen along the $\mathrm{C}^{2}-\mathrm{C}^{3}$ bond, and the $x$-axis is set toward the $\mathrm{H}^{4}$ atom at a right angle to the $z$-axis. 


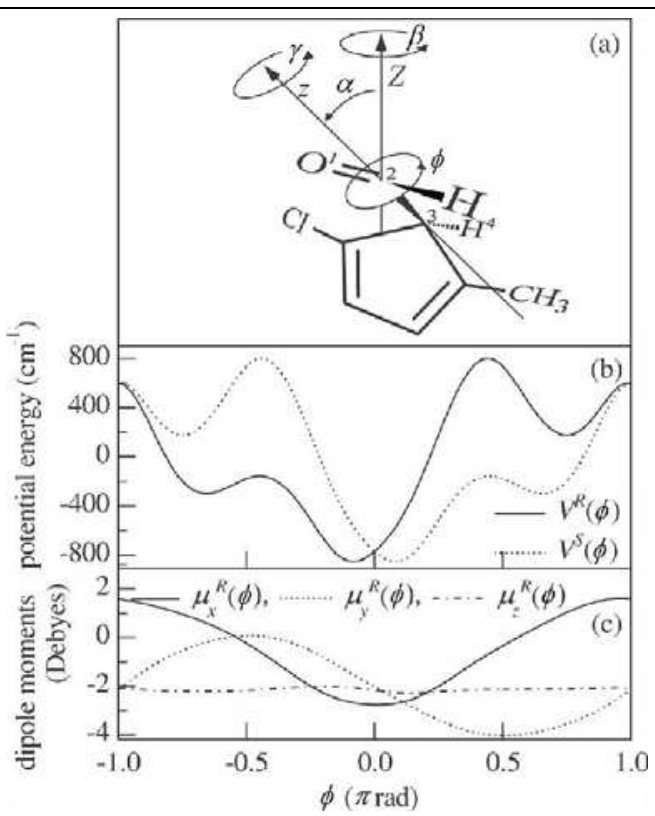

Fig. 3. (a) (R)-2-chloro-5-methylcyclopenta-2,4-dienecarbaldehyde as a chiral molecular motor. The aldehyde (-CHO) group is an motor driven by a laser pulse. (b) The ground state potential of $(R)$ - and $(S)$ - motors as a function of dihedral angle $\phi$. (c) Cartesian components of the dipole moment vector in the molecular frame $(x, y, z)$. Reproduced with permission from J. Chem. Phys., 119, 12393 (2003).

Consider a quantum dynamics of the motor in the electronic ground state since the frequency of the laser used is in the far-IR range. The model system has the following essential properties: (i) owing to molecular chirality, the potential energy function $V^{\Gamma}(\phi)$ is asymmetric such that $V^{S}(\phi-c) \neq V^{S}(-\phi+c)$ for any $c$, (ii) there is angle- $\phi$ dependency of the dipole moment vector because of the sufficient electronegativity of the $\mathrm{O}^{1}$ atom, and (iii) because the rotational constants of the molecule are large enough, the effects of the entire rotation of the molecule can be ignored. Therefore, the molecule interacting with a linearly polarized electric-field component of laser pulses can be a realistic model of the periodically perturbed ratchet system.

Molecular parameters were calculated by using the GAUSSIAN 98 package of programs (Frisch et al., 1998) with the 6-31G* basis set and the MP2 method. The moment of inertia $I$ is estimated as 17.6 amu $\AA^{2}$. The rotational constants are $1.97,1.16$ and $0.79 \mathrm{GHz}$, the time scale of which is sufficiently large to safely ignore the rotational effects of the entire molecule, or treat them classically. The potential energy curves for $(R)$ - and $(S)$-motors shown in Fig. 3b were evaluated at each dihedral angle $\phi$, where all the other structural parameters were optimized. It can be seen that these potential curves are asymmetric with respect to $\phi$ and are mirror images of each other. As can be seen from the optimized structure for the $(R)$ motor, the rotation in the negative direction of $\phi$ has a gentle slope compared to that of the positive direction and vice versa in the relation for the (S)-motor. These are well-known properties of a chiral molecule. 
Figure $3 \mathrm{c}$ shows three computed Cartesian components of the dipole moment of the $(R)$ motor, $\mu_{x} \mathrm{R}, \mu_{y} \mathrm{R}$ and $\mu_{z} \mathrm{R}$ as a function of $\phi$. If a rigid dipole moment rotates in $x y$-plane, then these functions can be expressed in the form of sinusoidal functions as

$$
\boldsymbol{\mu}^{\Gamma}(\phi)=\mu^{\Gamma} \cos (\phi) \boldsymbol{e}_{x}+\mu^{\Gamma} \sin (\phi) \boldsymbol{e}_{y}+\boldsymbol{\mu}_{0}^{\Gamma},
$$

where $\mu^{\Gamma}$ and $\mu_{0}^{\Gamma}$ are constants. It can be seen from Fig. $3 c$ that this is a fairly good approximation and $\mu^{R}$ is about -2.0 Debye. Therefore, the interaction energy with electric fields of a few $\mathrm{GVm}^{-1}$ is comparable to the internal rotational barrier height, $\Delta V=1650 \mathrm{~cm}^{-1}$. Assuming an electric field of a $z$-polarized laser pulse as

$$
\boldsymbol{E}(\mathrm{t})=f(\mathrm{t}) \cos (\omega \mathrm{t}) \boldsymbol{e}_{\mathrm{Z}},
$$

the effective potential in Eq. (1) becomes

$$
\mathrm{V}^{\Gamma}(\phi)-\boldsymbol{\mu}^{\Gamma}(\phi) \cdot \boldsymbol{E}(\mathrm{t})=\mathrm{V}^{\Gamma}(\phi)-f(\mathrm{t}) \mu^{\Gamma} \sin (\alpha) \cos (\phi+\gamma) \cos \omega \mathrm{t}-\boldsymbol{\mu}_{0}^{\Gamma} \cdot \boldsymbol{E}(\mathrm{t}) .
$$

Here, $\alpha$ and $\gamma$ are the Euler angles between the molecular frame $x y z$ and the laboratory frame $X Y Z$. The last term is a constant with respect to the variable $\phi$ and does not make any contribution to the dynamics of the molecular motor. The second term is the time-correlated periodic function of $\phi$, which rocks the potential energy function $V^{\Gamma}(\phi)$ as shown in the lower panels of Fig. 1.

Let us now consider the rotational dynamics including motor-bath interactions. Equation (3) can be reduced to a so-called Lindblad-type equation (Lindblad, 1976) by assuming the bath consists of harmonic oscillators and the motor-bath interaction is given in the first order of displacements for both bath motor coordinates as

$$
i \hbar \frac{\partial}{\partial t} \hat{\rho}^{\Gamma}(t)=\left[\hat{H}_{S}^{\Gamma}(t), \hat{\rho}^{\Gamma}(t)\right]+\frac{i}{2} \sum_{n}\left\{\left[\hat{A}_{n}^{\Gamma} \hat{\rho}^{\Gamma}(t), \hat{A}_{n}^{\Gamma \dagger}\right]+\left[\hat{A}_{n}^{\Gamma}, \hat{\rho}^{\Gamma}(t) \hat{A}_{n}^{\Gamma \dagger}\right]\right\},
$$

where $\hat{\rho}^{\Gamma}(t)$ is the reduced density operator of the motor and the system-bath coupling is phenomenologically introduced by the interaction operator $\hat{A}_{n}^{\Gamma}$.

We set the interaction operator $\hat{A}_{n}^{\Gamma}$ to $|n+1\rangle^{\Gamma} a_{n}^{\Gamma}\left\langle\left. n\right|^{\Gamma}+\mid n\right\rangle^{\Gamma} b_{n}^{\Gamma}\left\langle n+\left.1\right|^{\Gamma}\right.$. That is, only inelastic scattering processes were taken into account and elastic interactions were ignored. To semiquantitatively discuss relaxation effects, both the interaction parameters $a_{n}$ and $b_{n}$ were set to

$$
\begin{aligned}
& \frac{a_{n}^{2}}{b_{n}^{2}}=\exp \left(-\frac{E_{n+1}-E_{n}}{k T}\right), \\
& a_{n}^{2}+b_{n}^{2}=\frac{\hbar}{\tau_{0}} \frac{E_{n+1}-E_{0}}{E_{1}-E_{0}},
\end{aligned}
$$

where $E_{n}$ denotes the $n$th eigenstate of the motor in the ground state, and $\tau_{0}$ is relaxation time from $n=1$ to 0 . Condition (8a) assures detailed balance of the system at temperature $T$, and condition ( $8 \mathrm{~b})$ specifies the relaxation time such that the more the system is excited, the faster the energy relaxes. The quantum master equation, Eq. (7), was solved by means of the 
split operator and a finite difference method with a fast Fourier transform algorithm using 256 grids for $\phi$.

In order to discuss the dynamics of molecular motors in a quantum mechanical way, we now introduce an angular momentum operator for the internal rotation $-i \hbar(\partial / \partial \phi)$. An expectation value of the angular momentum operator at time $t$ in the coordinate representation is defined as

$$
\ell^{\Gamma}(t)=\int_{-\pi}^{\pi} d \phi^{\prime} \int_{-\pi}^{\pi} d \phi\left\{-i \hbar \frac{\partial \delta\left(\phi^{\prime}-\phi\right)}{\partial \phi} \rho^{\Gamma}\left(\phi, \phi^{\prime} ; t\right)\right\} .
$$

The instantaneous angular momentum of an ensemble of randomly oriented motors, averaged over all of the Euler angles $\Omega(\alpha, \beta, \gamma),\left\langle\ell^{\Gamma}(t)\right\rangle_{\Omega}$,

$$
\left\langle\ell^{\Gamma}(t)\right\rangle_{\Omega}=\frac{1}{8 \pi^{2}} \int \ell^{\Gamma}(t) d \Omega
$$

was calculated as an index of rotations of motors. The above integration was computed by the trapezoidal rule with 16 grid points for $\alpha$ and 64 grid points for $\gamma$.

Let us apply a linearly polarized electric field, Eq. (5) with the envelop function $f(t)$,

$$
f(t)=\left\{\begin{array}{cc}
E_{0} \sin ^{2} \frac{\pi t}{t_{p}} & \left(0 \leq t<t_{p}\right) \\
0 & \left(t<0, t_{p} \leq t\right)
\end{array} .\right.
$$

Here, the pulse length $t_{p}$ is 30 ps and the central frequency $\omega$ is $124 \mathrm{~cm}^{-1}$. The frequency is related to the frequency of a normal vibration which localizes to the torsion of the aldehyde group. The maximum amplitude of the electric field $E_{0}$ is set to $3.4 \mathrm{GVm}^{-1}$. Thus, the maximum difference of the dipole interaction energy, which is $2 \mu^{\Gamma} E_{0}$, is larger than the barrier height of the potential energy $\Delta V \approx 1,600 \mathrm{~cm}^{-1}$, and the wave packet of the motor can overcome the barrier even if the frequency of the laser is non-resonant to anharmonic potential energy function $V^{\Gamma}(\phi)$.

Figure 4 shows the time evolution of instantaneous angular momentum of randomly oriented molecular motors with initial density being set to the Boltzmann distribution at $T=150 \mathrm{~K}$. The axis on the left-hand side denotes angular momentum in units of $\hbar$, and the axis on the right-hand side denotes angular frequency in units of Hz. In Fig. 4, we obtained $\left\langle\ell^{R}(t)\right\rangle_{\Omega} \approx-1.6 \hbar$ for $(R)$-motors and $\left\langle\ell^{S}(t)\right\rangle_{\Omega} \approx 1.6 \hbar$ for $(S)$-motors after application of the laser pulse. In the absence of a relaxation process $\left(\tau_{0}=\infty\right)$ as a special case, $\left\langle\ell^{\Gamma}(t)\right\rangle_{\Omega}$ reaches the maximum value at around the pulse peak and maintains a certain value even after the pulse is turned off. This means that the motion created is not a pendulum one but a unidirectional rotation across the potential barrier. It should also be noted that rotational directions of randomly oriented motors are opposite in $(R)$ - and $(S)$-motors. If molecules do not have chirality, the angular momentum becomes zero. Here, an achiral motor is obtained by substituting a methyl group of the motor with a chlorine atom and its potential becomes symmetric as $V^{\mathrm{R}}(\phi)=V^{\mathrm{S}}(\phi)$. 


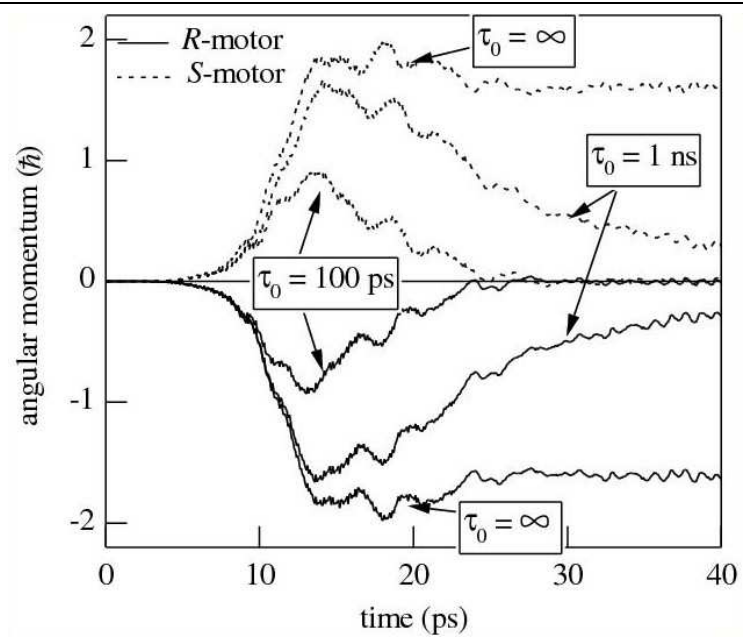

Fig. 4. Temporal behaviours of the instantaneous angular momentum of randomly oriented (R)-motors (solid lines) and those of (S)-motors (dotted lines) at $\mathrm{T}=150 \mathrm{~K} . \tau_{0}$ denotes the relaxation time the first excited vibrational state to the ground state. Reproduced with permission from J. Chem. Phys., 119, 12393(2003).

Figures 4 also shows the relaxation effects on $\left\langle\ell^{\Gamma}(t)\right\rangle_{\Omega}$. The relaxation time $\tau_{0}$ from the first excited vibrational state to the ground state is taken as a relaxation parameter. In the simulation, we assumed the system is weakly fluctuating by neglecting ultrafast inertial effects. Such a condition can be realized considering that the molecules are surrounded by solvent cage molecules or imbedded in rigid solvents under low temperature conditions. Strong $\tau_{0}$ dependence can be seen in Fig. 4, which is mainly due to the assumption that effective relaxation times were taken to be proportional to $\Delta V$, being shorter than that of $\tau_{0}$ by about two orders. One of the possibilities to drive rotation continuously is to supply laser pulses repeatedly to recover the loss of energies and accelerate the rotation.

Figure 5 shows the effective potential and rotational wave packets of the $(R)$-motor at several specific Euler configurations to qualitatively understand its unidirectional motion. The Euler angle $\alpha$ was fixed at $0.5 \pi$ to give the maximum interaction between the dipole moment vector and the photon polarization vector. The amplitude of the electric field was the same as that in Fig. 4. The rotational wave packet was initially localized around the stable configuration, $\phi \approx 0$. It can be seen from Fig. 5 that the initial rotational wave packet at the configuration $\gamma=0$ at $\omega t=\pi$ or $\gamma=\pi$ at $\omega t=0$ moves toward the left-hand side with a gentle slope when the wave packet is rocked by a strong pulsed laser. On the other hand, Figs. $5 \mathrm{c}$ and $5 \mathrm{~d}$ show that the rotational wave packet rocked by a pulsed laser at the configuration $\gamma= \pm 0.5 \pi$ cannot obtain sufficient angular momentum to cross the potential barrier after the laser pulse is turned off. The origin of the unidirectional motion is the asymmetry of the rotational potential. The linearly polarized laser pulse acts as a kick to the molecular motor. The initial angular momentum is large enough to determine the rotation toward the gentle slope of the rotational potential after the application of laser pulse. 


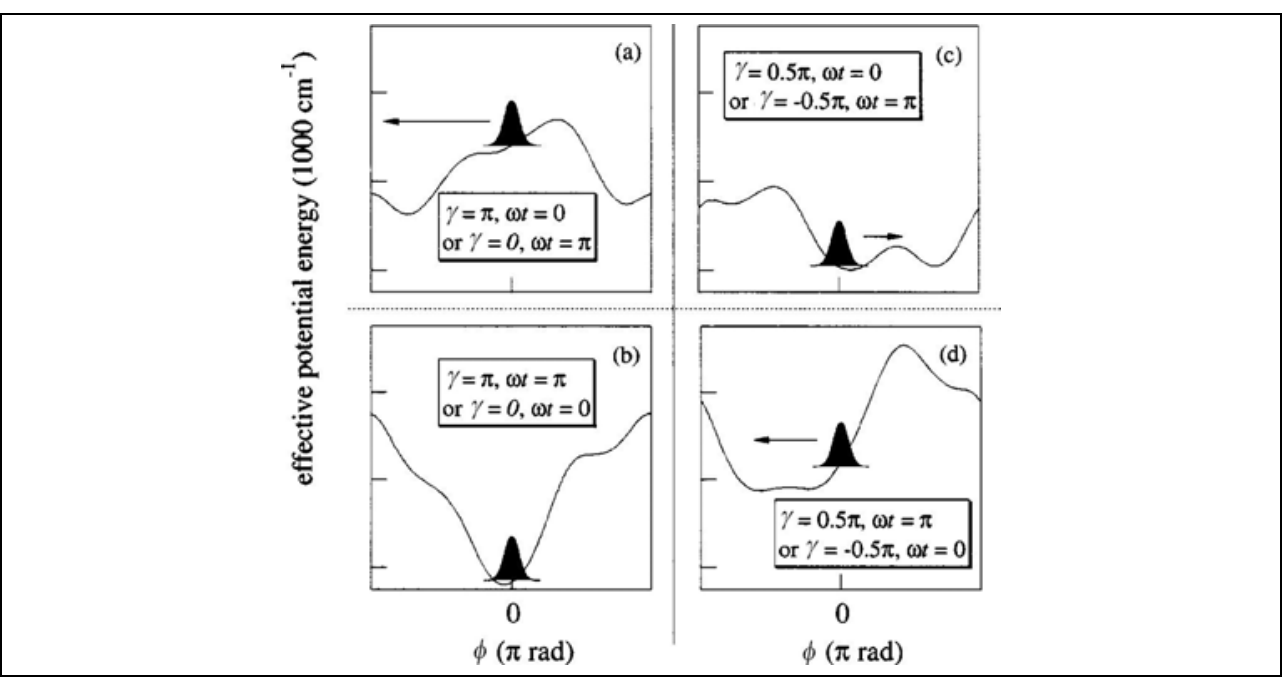

Fig. 5. A schematic view of a localized torsional wave packet on the effective potential of an $(R)$-motor fixed at a specific Euler angle $\gamma$ and time $\omega t$. The effective potential is constructed from the torsional potential and time-dependent periodic interaction between the chiral motor and laser pulse. Reproduced with permission from J. Chem. Phys., 119, 12393(2003).

\subsection{Femtosecond pump-dump laser-ignition method}

In this subsection, we describe another method called the pump-dump laser-ignition method for driving unidirectional motions of chiral molecular motors (Hoki et al., 2004). Figure 6 shows the control scheme. A femtosecond vis/UV pump pulse denoted by (1) creates a wave packet in an electronic excited state $V_{g}^{\Gamma}$. When the wave packet moves along the potential energy surface (PES) and reaches an appropriate position, it is dumped to the electronic ground state $V_{g}^{\Gamma}$ by applying the second pulse (2). In the dumping process, the kinetic energy of the rotary motion is conserved before and after the dumping process as a result of the Franck-Condon principle. Therefore, the pump-dump laser excitation can ignite a unidirectional motion of the rotational wave packet because the wave packet has a certain kinetic energy to overcome its activation barrier height in the ground state $V_{g}^{\Gamma}$. Because the electronic state is altered as $V_{g}^{\Gamma} \rightarrow V_{e}^{\Gamma} \rightarrow V_{g}^{\Gamma}$, by the pump-dump pulse sequences, this mechanism has an analogy to that of the flushing ratchet system. However, in the pump-dump laser-ignition method, quantum dynamics of wave packets of chiral motors are utilized for determining unidirectional motions as shown below.

The rotational direction is characteristic of the chirality of the molecular rotor. As shown in Fig. 6, the direction is determined by the gradient of $V_{\mathrm{e}}^{\Gamma}$ around its Franck-Condon region, and the gradient can be estimated using quantum chemistry calculations. We call this motion in the direction of rotation a regular rotation. If the molecule has a symmetric plane as in the case of an achiral molecule, the gradient should be zero. Therefore, an achiral molecule cannot produce unidirectional motion using a linearly polarized laser. As shown in Fig. 6, on the other hand, from symmetry considerations, the gradient has opposite signs between the $(R)$ - and $(S)$ - motors. 


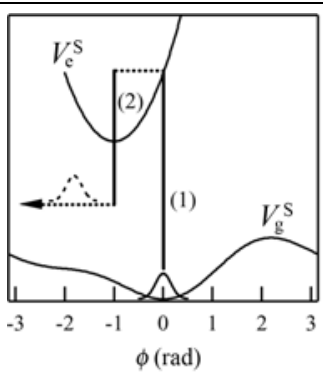

$\underline{\text { Case } 1}$

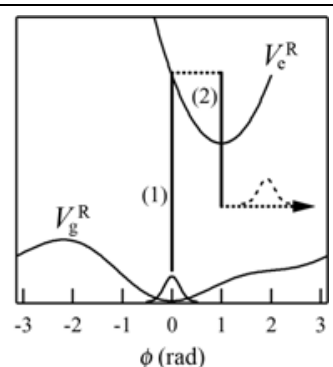

Case 2

Fig. 6. Scheme for a pump-dump laser-ignition: an (S)-motor (left) and an (R)-motor (right).

The time evolution of the molecular motor is determined by the time-dependent Schrödinger equation within the two-electronic state (the electronic ground and excited states) model

$$
i \hbar \frac{\partial}{\partial t}\left(\begin{array}{l}
\psi_{g}(\phi ; t) \\
\psi_{e}(\phi ; t)
\end{array}\right)=\left(\begin{array}{cc}
\hat{T}+V_{g}(\phi) & -\boldsymbol{\mu}_{g e}(\phi) \cdot E(t) \\
-\boldsymbol{\mu}_{e g}(\phi) \cdot \boldsymbol{E}(t) & \hat{T}+V_{e}(\phi)
\end{array}\right)\left(\begin{array}{c}
\psi_{g}(\phi ; t) \\
\psi_{e}(\phi ; t)
\end{array}\right)
$$

where $\boldsymbol{\mu}_{g e}(\phi)\left(=\boldsymbol{\mu}_{\mathrm{gg}}(\phi)\right)$ is the transition dipole moment between the two states, $V_{n}(\phi)$ is the potential energy in the electronic state $n(n=g$ and $n=e)$ and $\hat{T}=-\frac{\hbar^{2}}{2 I} \frac{\partial^{2}}{\partial \phi^{2}}$ with moment of inertia $I$ was assumed to be common in both electronic states. $E(t)$ is the electric field of vis/UV laser. $\psi_{n}(\phi ; \mathrm{t})$ is the nuclear wave packet.

The electric field of the linearly polarized pump laser with central angular frequency $\omega_{\mathrm{p}}$ and the linearly polarized dump laser with $\omega_{\mathrm{d}}$ is expressed as

$$
\boldsymbol{E}(t)=A_{p} f\left(t ; t_{p}, t_{p}+T_{p}\right) \cos \left(\omega_{p} t\right) \boldsymbol{e}_{\mathrm{Z}}+A_{d} f\left(t ; t_{d}, t_{d}+T_{d}\right) \cos \left(\omega_{d} t\right) \boldsymbol{e}_{\mathrm{Z}}
$$

with the pulse amplitude $A_{j}(j=\mathrm{p}$ and $\mathrm{d})$, the pulse duration $T_{j}$; the time delay $t_{j} . f(t)$ denotes the envelope function of the pulse,

$$
f\left(t ; t_{1}, t_{2}\right)=\left\{\begin{array}{cc}
\sin ^{2} \pi \frac{t-t_{1}}{t_{2}-t_{1}} & \left(t_{1} \leq t<t_{2}\right) \\
0 & \left(t<t_{1}, t_{2} \leq t\right)
\end{array} .\right.
$$

In order to estimate the magnitude of rotary motion of the motor, quantum-mechanical expectation value of the angular momentum in the electronic ground state $\ell_{g}(t)$, defined as

$$
\ell_{\mathrm{g}}(\mathrm{t})=\int_{-\pi}^{\pi} \mathrm{d} \phi \psi_{\mathrm{g}}^{*}(\phi ; \mathrm{t})\left(-\mathrm{i} \hbar \frac{\partial}{\partial \phi}\right) \psi_{\mathrm{g}}(\phi ; \mathrm{t})
$$

was calculated. 
Figure 7 shows the result of ab initio calculation of (R)-2-methylcyclopenta-2,4 dienecarbaldehyde in $S_{0}$ and $S_{1}$. The fully relaxed geometrical structure of the molecule was calculated at the MP2/6-31+G** level of theory at each point of the chosen reaction coordinate $\phi$. The potential energy in the electronic ground-state $S_{0}$ and that in the first excited singlet state $S_{1}$ were calculated by means of multi-configuration methods (Ross, 1989).

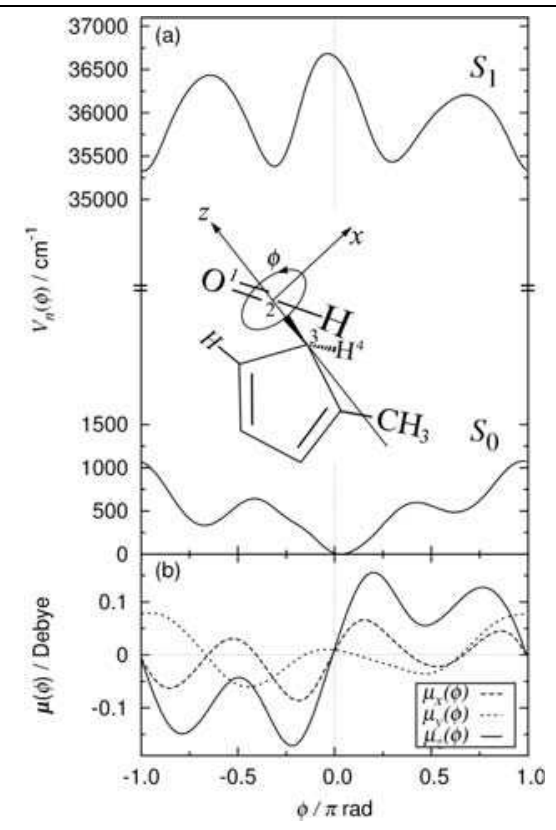

Fig. 7. (a) Potential energies in the ground and first excited singlet states of (R)-2-methylcyclopenta-2,4-dienecarbaldehyde. (b) Transition moments as a function of $\phi$. Reproduced with permission from J. Phys. Chem. B, 108, 4916 (2004).

Figure 7a shows the PESs of the $(R)$-motor in $S_{0}$ and $S_{1}$. Because the $S_{1}$ PES slope in the Franck-Condon region is negative, a wave packet created by a pump pulse would run with a positive angular momentum. Note that the minimum energy position in the ground state is slightly shifted in a positive direction. This is reflected in the geometry of the molecule by a slight bend of the aldehyde group toward the methyl group by about 7 degrees.

We now apply the pump-dump laser-ignition method to drive a rotation of the $-\mathrm{CHO}$ group of (R)-2-methyl-cyclopenta-2,4-dienecarbaldehyde. To analyze the rotational dynamics, we solved the time-dependent Schrödinger equation (12) by means of the splitoperator method with the help of an FFT algorithm using 256 grid points for $\phi$. Here, the motor-laser interaction, $-\mu_{g e}(\phi) \cdot E(t)$ was treated numerically using Pauli matrices (Choi, 1989).

The upper panels a, b, and c in Fig. 8 show the time evolution of the rotational wave packets created in $S_{0}$ by applying the pump-dump laser-ignition method. We omitted wave packets trapped in the $S_{0}$ potential well because they do not evolve after the dump pulse is turned off. Here, the parameters of pulses used were $A_{\mathrm{p}}=A_{\mathrm{d}}=10^{10} \mathrm{~V} / \mathrm{m}, T_{\mathrm{p}}=T_{\mathrm{d}}=100 \mathrm{fs}, t_{\mathrm{p}}=50 \mathrm{fs}$, 
$t_{\mathrm{d}}=180 \mathrm{fs}, \omega_{\mathrm{p}}=34,300 \mathrm{~cm}^{-1}$, and $\omega_{\mathrm{d}}=32,800 \mathrm{~cm}^{-1}$. The initial state was set on the lowest rotational level in the electronic ground state. It can be seen that in the early stage of the time evolution, the motor takes about $550 \mathrm{fs}$ for one cycle, which corresponds to angular frequency $\omega$ of $1.1 \times 10^{13} \mathrm{~s}^{-1}$. From an analogy to classical mechanics, $\ell \approx \omega I$ and the expectation value of the angular momentum of the motor is estimated to be $\ell \approx 31 \hbar$.

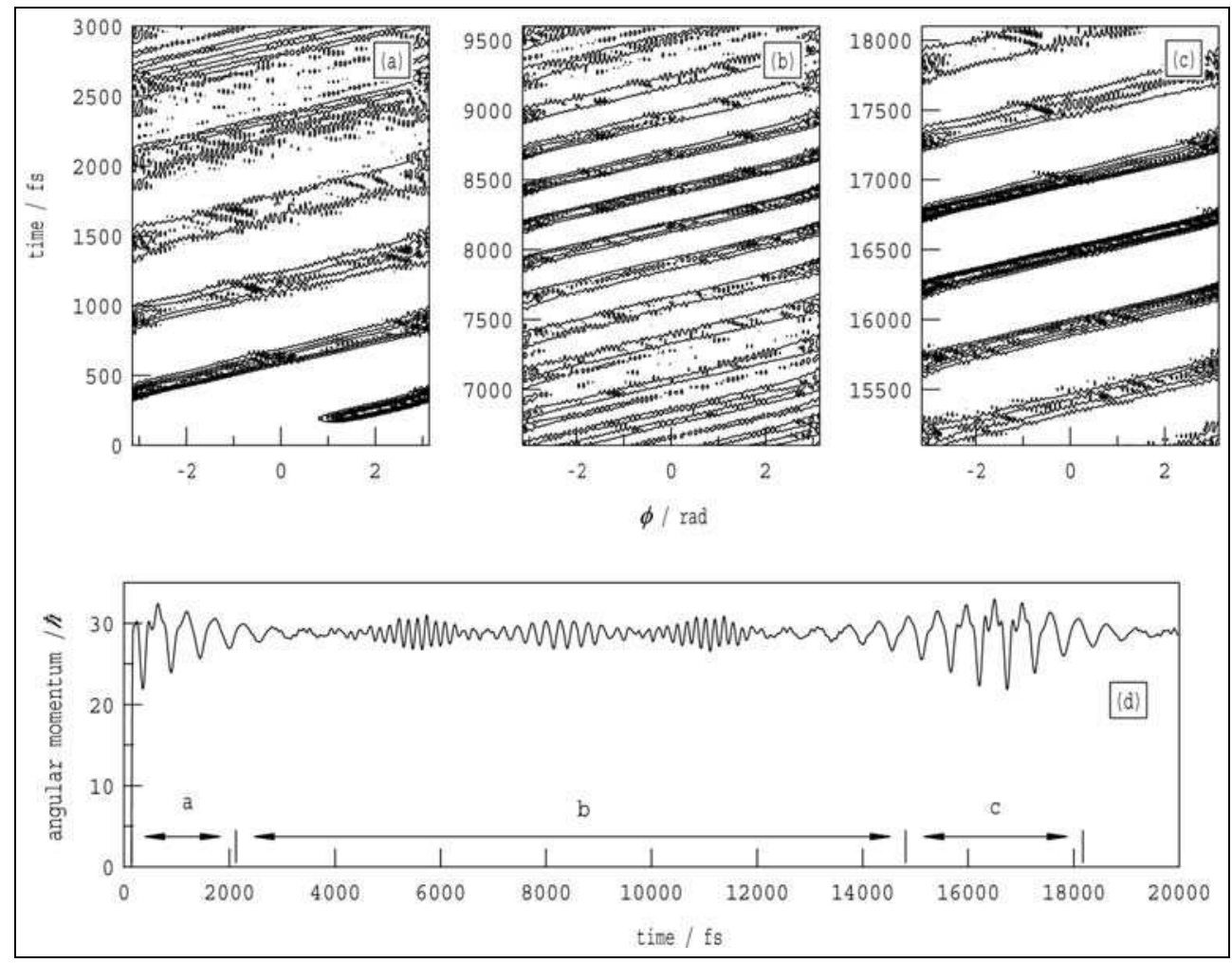

Fig. 8. Quantum dynamics behaviors of (R)-2-methyl-cyclopenta-2,4-dienecarbaldehyde, which were induced by a pump-dump laser-ignition method. Reproduced with permission from J. Phys. Chem. B, 108, 4916 (2004).

An interesting feature appears in panel $b$ where the rotational wave packet is split into two wave packets. These wave packets converge to a single one again after about 1,500 fs, as shown in panel c. Panel $d$ shows that the time-dependent expectation value of the angular momentum $\ell_{\mathrm{g}}(t)$ can be divided into three regions, a, b and $\mathrm{c}$, within rephrasing time of about $17,000 \mathrm{fs}$. These three regions are the same as those in the upper panels.

This feature can be explained by a free rotor model, where the eigenfunction is $1 / \sqrt{2 \pi} \exp (i m \phi)$ with eigenvalue $\varepsilon_{m}=\hbar^{2} m^{2} / 2 I$. The wave packet $\psi_{\mathrm{g}}(\phi, t)$ can be expressed as

$$
\psi_{g}(\phi ; t)=\frac{1}{\sqrt{2 \pi}} \sum_{m=m_{a}}^{m_{b}} C_{m} \exp (i m \phi) \exp \left(-\frac{i}{\hbar} \varepsilon_{m} t\right) .
$$


Here, the coefficient $C_{m}$ which represents the probability amplitude of the rotational quantum number $m$ is determined at the time when the dump pulse is applied. Under the condition of a localized distribution of the angular momentum, the wave packet at half of the rephrasing time, $\pi I / \hbar$, can be expressed (Hoki et al., 2004) as

$$
\psi_{g}(\phi ; t=\pi I / \hbar)=\frac{1}{\sqrt{2}} \exp \left[-\frac{i \pi}{4}\right] \psi_{g}(\phi ; t=0)+\frac{1}{\sqrt{2}} \exp \left[\frac{i \pi}{4}\right] \psi_{g}(\phi-\pi ; t=0)
$$

This expression indicates that the wave packet splits into two parts with the same magnitudes at half of the rephrasing time. The rephrasing time in the present motor system is roughly estimated to be $2 \pi I / \hbar=17,000 \mathrm{fs}$, which is the same order as the numerically evaluated one, as shown in panel c. This explains why the splitting of the rotational wave packet into two parts takes place in panel $b$.

\subsection{Method for detecting quantum dynamics of molecular motors in real time}

In the preceding subsections, we adopted a quantum-mechanical expectation value of angular momentum to theoretically prove rotary motions of chiral molecular motors. However, it is not so simple to experimentally detect the expectation values of angular momenta of neutral molecular motors by using optical or magnetic methods. In this subsection, we briefly refer to observation of ultrafast motions of molecular motors in real time. There are several spectroscopic methods for observation of nuclear motions on reaction potential surfaces in the field of so-called femtosecond chemistry (Potter et al., 1992; Zewail \& Bernstein, 1992). In this subsection, we adopt a time- and frequency-resolved ionization method. We show by using a time-frequency-resolved ionization simulation that the results obtained in Sec. 2.3 can be experimentally observed. The method consists of a femtosecond probe pulse of central frequency $\omega_{3}$ for ionization of molecular motors in a nonstationary state in addition to pump and dump pulses. Ionization signals are plotted as a function of $\omega_{3}$ after fixing a delay time $t_{3}$ between the dump and probe pulses.

Figure 9 shows calculated time-frequency-resolved spectra $I\left(\omega_{3}, t_{3}\right)$ of $(R)$-2-methylcyclopenta-2,4-dienecarbaldehyde (Hoki et al., 2004). The pulse duration was set to $100 \mathrm{fs,}$ and the maximum value of the pulse amplitude was set to a small value $\left(10^{7} \mathrm{Vm}^{-1}\right)$ in order to satisfy a one-photon ionization in a weak field limit. We can see an oscillatory behavior of the spectrum at $\omega_{3}=60,900 \mathrm{~cm}^{-1}$ corresponding to the ionization potential around $\phi=0$. Figure 9 shows wave packet motions of the molecular motor in the ground state. The time evolution of the intensity peaks reflects the energy of the ionization potential as a function of $\phi$ (Hoki et al., 2004). The time for one cycle oscillation was estimated to be $540 \mathrm{fs}$ since two peaks of the oscillation are located at 380 and $920 \mathrm{fs}$.

The method for the time- and frequency-resolved spectrum described above can be applied to detect molecular motor dynamics not only for pump-dump laser-ignition method but also those for other methods described in this chapter.

\section{Optimal control of unidirectional motions}

There are two types of theoretical methods for designing optimal laser pulses. One is a local control method and the other is a global control method (Gordon \& Fujimura, 2002). Here, "local" means that maximization of the target is carried out at each time. Therefore, the 


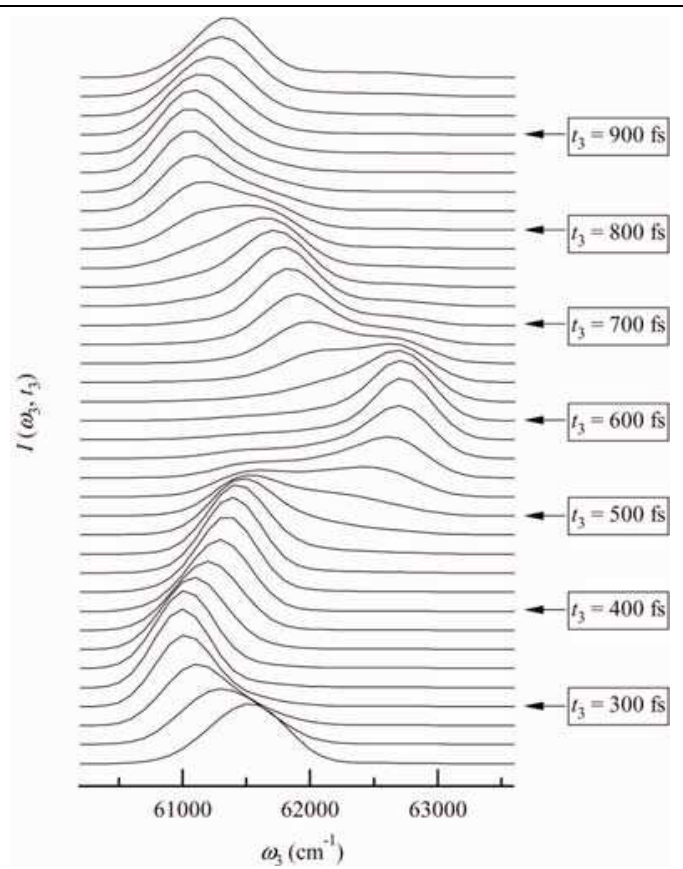

Fig. 9. Time-frequency-resolved spectra $I\left(\omega_{3}, t_{3}\right)$ of $(R)$-2-methyl-cyclopenta-2,4dienecarbaldehyde. Reproduced with permission from J. Phys. Chem. B, 108, 4916 (2004).

algorithm is quite simple, and only one-sided propagation, forward or backward propagation, is needed to solve the time-dependent Schrödinger equation. A global control method is, on the other hand, general and both forward and backward propagations have to be carried out. However, it can be applied to any dynamical systems, though sometimes converging problems arise.

\subsection{Local control of a molecular motor}

In the local control method, electric field of laser pulses $\boldsymbol{E}(t)$ is given as

$$
\boldsymbol{E}(t)=-2 A \operatorname{Im}\langle\Psi(t)|\hat{W} \hat{\boldsymbol{\mu}}| \Psi(t)\rangle
$$

where $A$ is a regulation parameter of the laser intensity, and $\hat{W}$ is the target operator. Substituting $\left|\Psi\left(t_{0}\right)\right\rangle=|0\rangle$ into Eq. (18), we obtain an electric field $E\left(t_{0}\right)$ using Eq. (18) at the initial time $t_{0}$. Solving the time-dependent Schrödinger equation with the initial condition of $\left|\Psi\left(t_{0}\right)\right\rangle$, we obtain a wave function after an infinitesimally increased propagation time. With this form, the procedure described above is repeated until $E\left(t_{f}\right)$ at the final time $t_{f}$ is obtained. The locally optimized electric field $E(t)$ guarantees a monotonic increase in the expectation value of the target operator $\hat{W}$ if it commutes with the molecular Hamiltonian, i.e., $\left[\hat{H}_{0}, \hat{W}\right]=0$. This condition can be satisfied when the target operator has the form of $\hat{W}=\sum|n\rangle w_{n}\langle n|$, where $|n\rangle$ is an eigenstate of $\hat{H}_{0}$ and $w_{n}$ is a waiting factor. 
Consider a quantum control which yields a unidirectional rotational state $|T\rangle$ from the ground state $|0\rangle$. Unfortunately, we cannot set the target operator proportional to a projector $|T\rangle\langle T|$ because the projector does not commute with the Hamiltonian $\hat{H}_{0}$. To overcome this difficulty, we make use of time-reversal symmetry of the time-dependent Schrödinger equation. We design the locally optimal electric field $E(t)$ by carrying out backward propagation starting from the target state $|T\rangle$ to yield the initial state $|0\rangle$ as much as possible. By doing so, we can set our target as the form of $\sum|n\rangle w_{n}\langle n|$, where the waiting factor $w_{n}$ should satisfy condition $w_{n-1}>w_{n}$ to ensure sequential population transfer to the ground state $|0\rangle$. Once the optimized electric field is determined, the motor dynamics is evaluated by solving the time-dependent Schrödinger equation (18) in the forward propagation.

We now apply the local control procedure described above to $(R)$-2-chloro-5-methylcyclopenta-2,4-dienecarbaldehyde. We construct the target state by using two eigenstates as $|T\rangle=(|66\rangle+i|65\rangle) / \sqrt{2}$ for a counter-intuitive rotation toward the steep slope of the potential energy curve and $|T\rangle=(|66\rangle-i|65\rangle) / \sqrt{2}$ for an intuitive rotation toward the gentle slope of the potential energy curve. These two eigenstates are chosen because (i) the eigen energy of the 65th and 66th molecular states is higher than the potential barrier of $1,500 \mathrm{~cm}^{-1}$ and (ii) the energy difference is less than $0.001 \mathrm{~cm}^{-1}$, so that these states practically degenerate in our observation time and correspond to the quantum number of $m= \pm 33$ of a free rotation system.

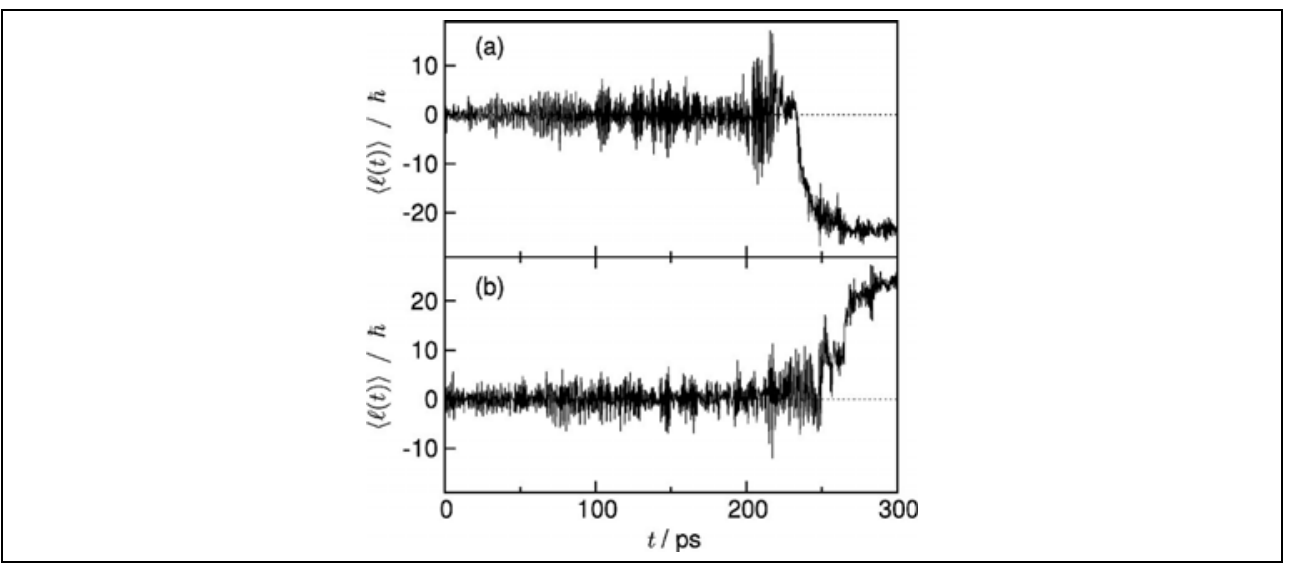

Fig. 10. (a) Instantaneous angular momentum of the (R)-motor driven by a local control method for the intuitive rotation and (b) for the counter-intuitive rotation. Reproduction with permission from Phys. Chem. Chem. Phys., 7, 1900 (2005).

Figure 10 shows time-dependent behaviors of the instantaneous angular momentum $\ell(t)$ of the $(R)$-motor molecule defined in Eq. (15), where the direction of the linearly polarized electric field vector is set to the $x$-axis. This clearly shows that rotational directions of the motor are controlled well, i.e., at the final time of 300 ps, the instantaneous angular momentum becomes constant values of about $-23 \hbar$ and $23 \hbar$ in Fig. 10a and 10b, respectively. It should be noted that there exists a time difference in the initiation between the intuitive and counter-intuitive rotational directions. This is discussed by using the Fig. 11. 
The figure in the left-hand side of Fig. 11 shows time- and frequency-resolved spectra of the locally optimized electric fields, $S(\omega, t)$, to rotate the motor in the intuitive and counter-intuitive directions shown in Fig. 10. A time- and frequency-resolved spectrum is defined as

$$
S(\omega, t)=\left|\int d \tau E(\tau) g(t-\tau)\right|^{2},
$$

where $g(t)$ is a window function. We can see that the electric fields consist of four components $\left(\varepsilon_{1}, \varepsilon_{2}, \varepsilon_{3}\right.$ and $\left.\varepsilon_{4}\right)$. The first two components, $\varepsilon_{1}$ and $\varepsilon_{2}$, simultaneously operate at the initial stage of motor initiation (0-160 ps). The third component, $e_{3}$, dominates in the low-frequency regime of the rotary motion whose potential is highly anharmonic. The third component bridges between the initial stage and the final stage of initiation (180-250 ps) at which unidirectional rotation starts. The fourth component, $\varepsilon_{4}$, accelerates the rotary motion. The frequency of this component is around $60 \mathrm{~cm}^{-1}$, which is close to the frequency difference between two quasi-degenerate pairs $n=63,64$ and $n=65,66$.

Two features appear in the initial two components $\varepsilon_{1}$ and $\varepsilon_{2}$. One feature is that $\varepsilon_{1}$ consists of a central frequency of about $60 \mathrm{~cm}^{-1}$ and $\varepsilon_{2}$ consists of a central frequency of $120 \mathrm{~cm}^{-1}$; that is, the latter is twice the former. This feature reflects optical transitions between eigenstates of a chiral molecule: the dipole moment of the chiral molecule is proportional to $\cos \phi$ and the transition moment between the $k$ th and $l$ th eigenstates involves both odd and even quantum transitions since the minimum of the asymmetric potential energy function is slightly shifted in the minus direction as can be seen in Fig. $3 b$ because of the molecular chirality. The other feature is that the frequencies in both components are expressed by a negative chirp behavior. The negative chirp form of the electric fields originates from a gradual decrease in the frequency difference between two transitions with $n=0-16$.

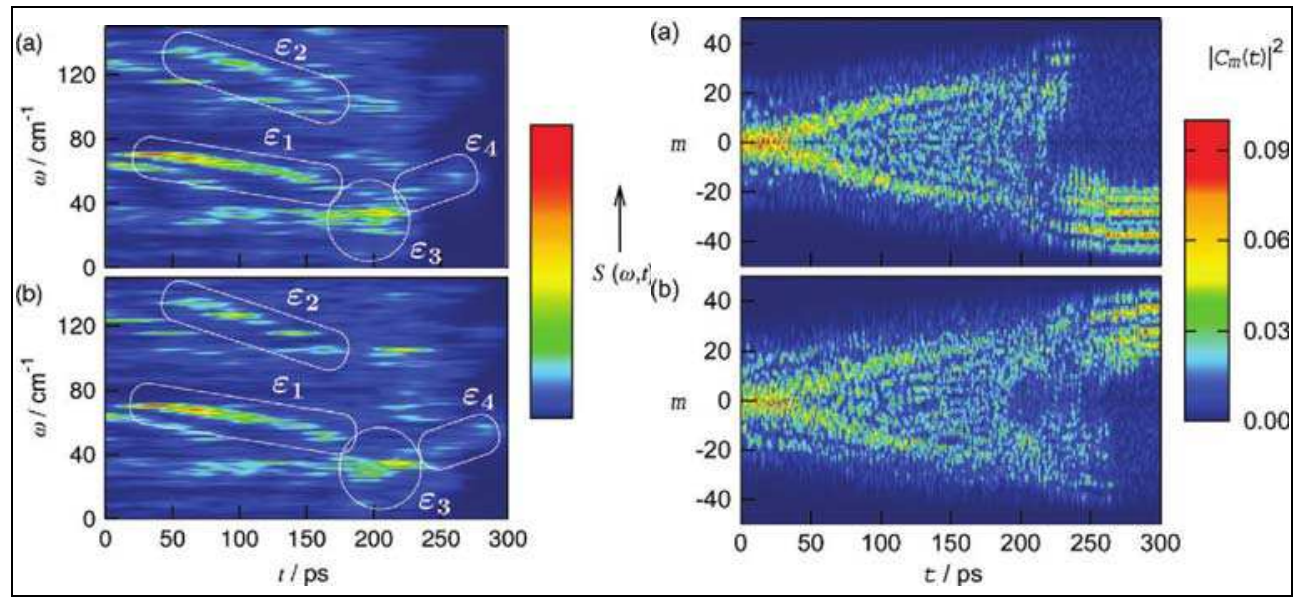

Fig. 11. (Left) Time- and frequency-resolved spectra of the locally optimized electric fields, $S(\omega, t)$, for (a) intuitive and (b) counter-intuitive directions. (Right) Time-dependent populations under the designed electric field $E_{x}(t)$ for (a) intuitive and (b) counter-intuitive directions. Reproduced with permission from Phys. Chem. Chem. Phys., 7, 1900 (2005). 
To clarify the mechanism of unidirectional rotation under the condition of irradiation of controlled laser pulses, we examined when unidirectional motion begins. In the right-hand side of Fig. 11, time-dependent populations under the designed electric field $E_{x}(t)$ are shown. We can see that a bunch of eigenstates $\{|n\rangle\}$ make a significant contribution to creation of a linear combination to compose the unidirectional rotational state. This is mainly due to the effect of the potential of the chiral motor. The intuitive rotation begins at about $t=240 \mathrm{ps}$, while the counter-intuitive rotation begins at about $t=260 \mathrm{ps}$. The difference of $20 \mathrm{ps}$ between these two rotations corresponds to about $1.1 \mathrm{~cm}^{-1}$. This frequency is close to the difference in frequency between the two eigenstates $n=57$ and 58 . This indicates that the direction of rotation is determined by the phase of a coherent superposition of rotational eigenstates created by locally optimized electric fields. Therefore, the time to change the direction of rotation can be selected by timing of designed pulses.

We note in Fig. 11 that timing of the $\varepsilon_{3}$ pulse component is different between intuitive and counter-intuitive directions. This is the essential factor for determining the rotational direction. Figure 10 clearly shows that creation of coherent states, i.e., rotational states in the intuitive direction or those in the counter-intuitive direction, depends on the electric fields estimated by using local control laser fields. Note that the intuitive rotation is controlled earlier than the counter-intuitive rotation by $20 \mathrm{ps}$. The earlier control of the rotary motion in the intuitive direction is related to the fact that the intuitive rotation is induced when a non-optimized laser field is applied.

\subsection{Global control of a molecular motor via an excited state}

In this subsection, we present results of application of a global optimization method to designing femtosecond pulses for unidirectional rotary motions of a chiral motor through an excited state.

A global control method tailors an electric field $E(t)$ by solving an optimization problem of the objective functional

$$
Y[E(t)]=\left\langle\Psi\left(t_{f}\right)|\hat{W}| \Psi\left(t_{f}\right)\right\rangle-\int_{0}^{t_{f}} d t \frac{1}{\hbar A(t)}|E(t)|^{2},
$$

Here, $\hat{W}$ is a target operator, $t_{f}$ is control time and $A(t)$ is a regulation function for tuning laser intensity. Under the variation condition, $\delta Y / \delta E(t)=0$ for any $t$ in the range $\left[0, t_{f}\right]$, an expression for the optimal field $E(t)$ is obtained as

$$
E(t)=-A(t) \operatorname{Im}\langle\Xi(t)|\hat{\mu}| \Psi(t)\rangle,
$$

where $|\Xi(t)\rangle$ is the time-dependent Lagrange multiplier that is a backward solution of the time-dependent Schrödinger equation with the final condition $\left|\Xi\left(t_{f}\right)\right\rangle=\hat{W}\left|\Psi\left(t_{f}\right)\right\rangle$. The optimal field is obtained by solving both the time-dependent Schrödinger equation with the initial condition $|\Psi(t=0)\rangle=|0\rangle$ and that with the final condition.

Again, we consider (R)-2-methyl-cyclopenta-2,4-dienecarbaaldehyde as a chiral molecular motor. The target operator for a unidirectional rotation with a positive angular momentum in the ground state, $\hat{W}_{g}^{+}$, is given in terms of the angular momentum basis set $|m\rangle$ as

$$
\hat{W}_{g}^{+}=\sum_{m>0}|m\rangle w_{m}\langle m|,
$$


where $w_{m} \geq 0$ is a weighting factor. In a similar way, the target operator for a unidirectional rotation with a negative angular momentum is given as

$$
\hat{W}_{g}^{-}=\sum_{m<0}|m\rangle w_{m}\langle m|
$$

Here, we note that the target operator for the regular rotation of the motor in the ground state is given as Eq. (22a) since the momentum created at the Franck-Condon region is positive as we discussed in the Sec. 2.3. The target operator for the reverse rotation is given as Eq. (22b).

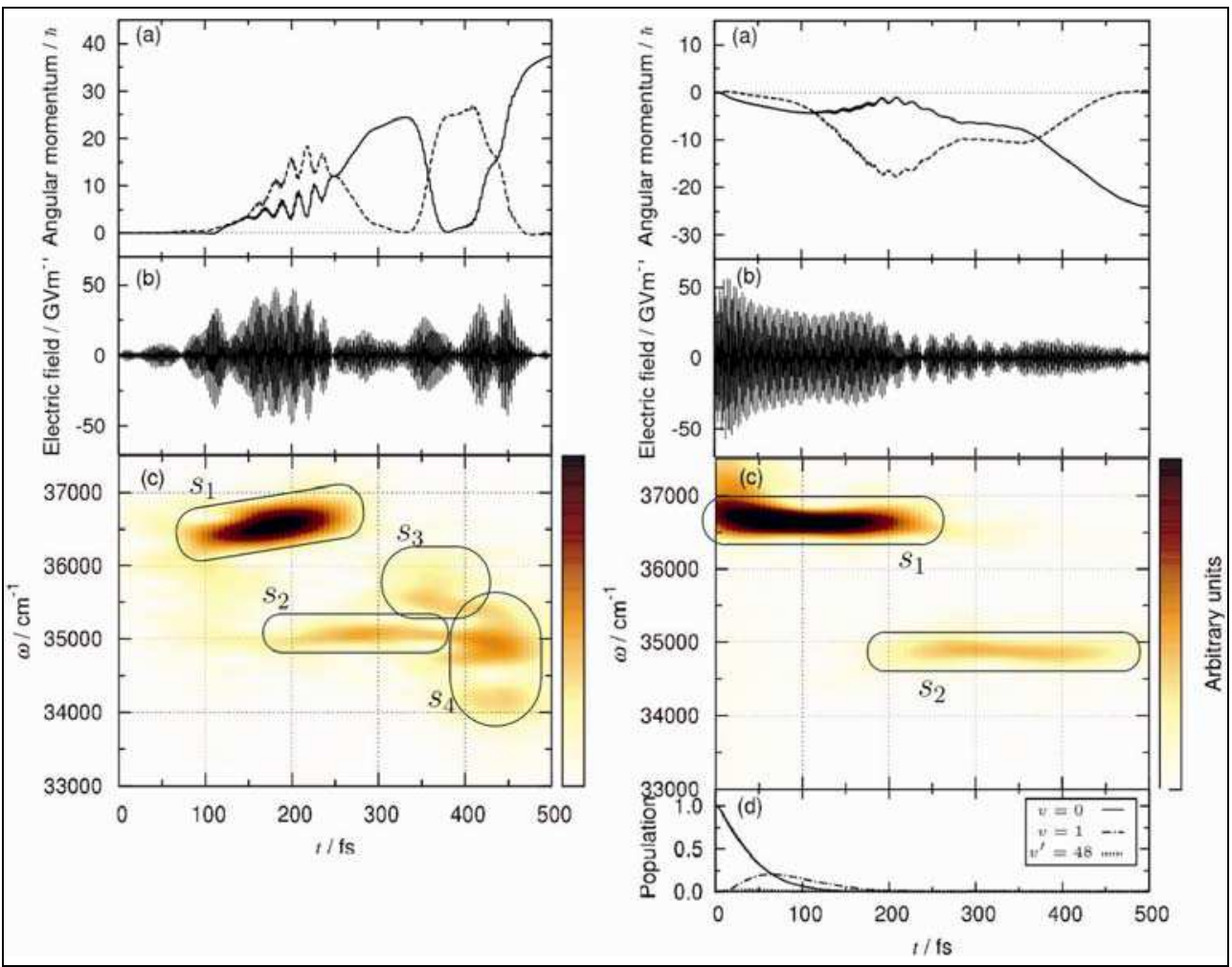

Fig. 12. (Left) Results for global control for driving (R)-2-methyl-cyclopenta-2,4-dienecarbaldehyde in the regular direction. (Right) Results for driving the motor in the reverse direction. Reproduced with permission from Chem. Phys., 347, (2008).

Figure 12a (left-hand side) shows the instantaneous angular momentum $\ell_{n}(t)(n=e$ or $g$ ) for the regular rotation which were obtained by using the optimal pulses in Fig. 12b. Here, the control time was set to $t_{f}=500 \mathrm{fs}$ and a $z$-polarized linear electric field was used. The solid line denotes the instantaneous angular momentum in the ground state $S_{0}, \ell_{g}(t)$, and the dashed line denotes that in the first electronic excited state $S_{1}, \ell_{e}(t)$. At the final time, $\ell_{g}\left(t_{f}\right) \approx 37 \hbar$ was obtained for the regular rotation. The angular momentum $\ell_{e}(t)$ increases 
faster than $\ell_{g}(t)$ in the early stage, $t<250 \mathrm{fs}$. This indicates that the direction of the regular rotation is determined in $S_{1}$ as explained in section 2.3.

Figure 12c shows the time- and frequency-resolved spectrum $S(\omega, t)$ for the optimal pulses in Fig. 12b. Here, the main components are labeled by $s_{1}, s_{2}, s_{3}$ and $s_{4}$. The first component for the regular rotation, $s_{1}$, with the central frequency of $36,400-36,800 \mathrm{~cm}^{-1}$ corresponds to the frequency range estimated from differences between $V_{e}(\phi)$ and $V_{g}(\phi)$. The feature of $s_{1}$ is a positive chirping that is the origin of the sharp increase in $\ell_{e}(t)$ between 100 and $250 \mathrm{fs}$ in Fig. 12a. The second component, $s_{2}$, has a central frequency around $35,000 \mathrm{~cm}^{-1}$, which corresponds to the energy difference between $V_{e}(\phi)$ and $V_{g}(\phi)$ at $\phi \approx 0.3 \pi$ as well. We note in Fig. 12c that the pulse around 100-250 fs has a positive chirp character to control motion of the excited wave packet that has a wide distribution of torsional states in $S_{1}$ with torsional quantum numbers $v^{\prime}=32-48$. Except for the appearance of the chirping element in the optimal pulse, the pulses for optimal control of the rotation of the motor in the positive direction are basically the same as those obtained by using the pump-dump laser-ignition method described in section 2.3.

Results for optimal control of the motor in the negative direction are shown in the righthand side of Fig. 12. At the final time, $\ell_{g}\left(t_{f}\right) \approx-24 \hbar$ was obtained, the magnitude $\left|\ell_{g}(t)\right|$ being small compared with that for the regular rotation. The optimal electric field reaches the maximum amplitude of $c a 50 \mathrm{GVm}^{-1}$ in the early stage, which is about the same intensity as that for the regular rotation. Also, the large amplitude is maintained longer duration than that for the regular rotation. These results indicate the complexity in control for reverse rotation compared with the results for regular rotation.

Figure 12c (right-hand side) shows the time- and frequency-resolved spectrum of the optimal field for the reverse rotation. It consists of two main components in the optimal pulses, labeled $s_{1}$ and $s_{2}$. The pulse $s_{1}$ with central frequency of $36,700 \mathrm{~cm}^{-1}$ causes a coherent emission to torsional quantum states in $S_{0}$ in the early stage, which is a coherent Stokes pulse. The other component $s_{2}$ is a dump pulse and creates a rotational wave packet in $S_{0}$.

Figure $12 \mathrm{~d}$ shows the time-dependent population of torsional eigenstates for the reverse rotation, $v=0,1$ in $S_{0}$ and $v^{\prime}=48$ in $S_{1}$. Here, $v^{\prime}=48$ is the dominant resonant state of the coherent Stokes scattering process. Figure $12 \mathrm{~d}$ indicates that a linear combination of $v=0$ and $v=1$ is created around $t=100 \mathrm{fs}$. Note that a quarter of the oscillation period of the superposition state is $130 \mathrm{fs}$, so that the superposition state in $S_{0}$ could obtain sufficient displacement to change the Franck-Condon excitation as shown in Fig. 7.

It should be noted that a new mechanism for the reverse rotation was found by applying a global control method. The direction of the rotational motion is determined by the coherent Stokes scattering processes via excited states above energy levels over the threshold in $S_{1}$.

\section{Laser-driven molecular machine}

\subsection{Model}

In this section, we present results of a quantum dynamics simulation of a laser-driven molecular machine, which is an extension of the chiral molecular motors discussed in the preceding sections. Here, we adopt a real molecule, $(R)$-2-chloro-5-trifluoromethylcyclopenta-2,4-dienecarbaldehyde ( $\left.\mathrm{cp}-\mathrm{Cl}_{-}-\mathrm{CF}_{3}-\mathrm{CHO}\right)$, as shown in Fig. 13. The molecule consists of three units: an aldehyde group $(-\mathrm{CHO})$, a trifluoromethyl group $\left(-\mathrm{CF}_{3}\right)$ and a 
cyclopentadiene with a chlorine atom as a main body. The aldehyde group can be driven by laser pulses, but the trifluoromethyl group cannot be because it is optically inactive. In that sense, the molecule can be regarded as one of the smallest molecular machines: the motion of the aldehyde group as a motor and that of the trifluoromethyl group as a gear or a propeller. We note that there is no belt or chain which directly connects the motor and blades of the gear in contrast to a macroscopic fan. It would be interesting to see if the machine can work by irradiation of laser pulses and to determine how power is transmitted from the motor to the running propeller and what the transmission mechanism is if it works.

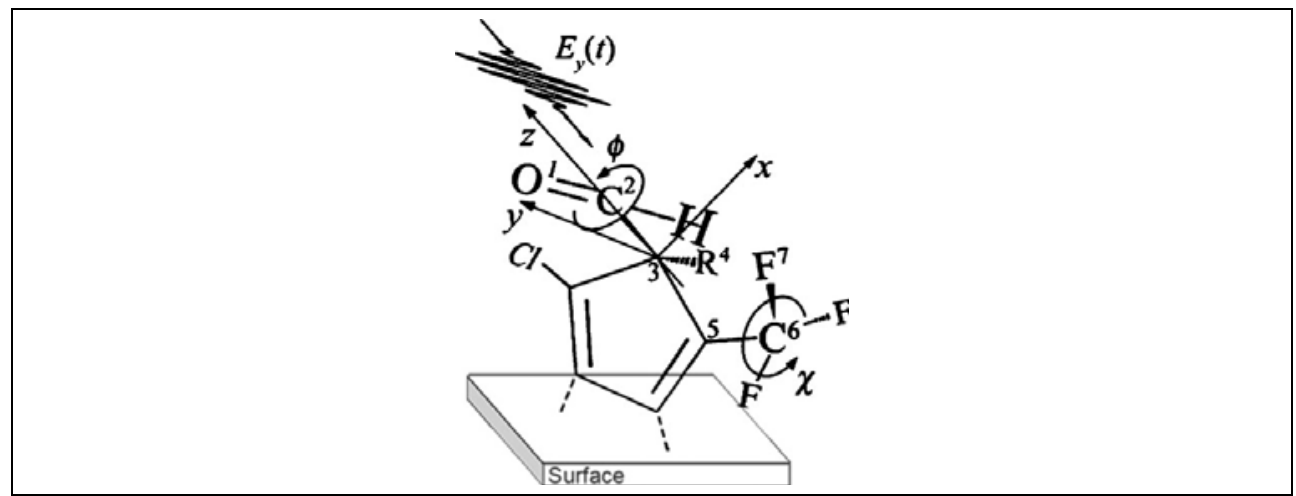

Fig. 13. (R)-2-chloro-5-trifluoromethyl-cyclopenta-2,4-dienecarbaldehyde attached at a surface as a molecular machine. The $\mathrm{C}^{3}$ atom is a chiral center. The $\mathrm{z}$-axis is defined to be along the $\mathrm{C}^{3}-\mathrm{C}^{2}$ bond. $\mathrm{R}^{4}$ denotes an alkyl group. A linearly polarized laser pulse propagating along the $y$-axis $E_{y}(t)$ is applied. A torsional coordinate of the aldehyde group is denoted by $\phi$ and that of the trifluoromethyl group is labeled by $\chi$. Reproduced with permission from Phys. Chem. Chem. Phys., 11, 1662 (2009).

For the sake of simplicity, we treat the quantum dynamics simulation of the molecular machine in a two-dimensional model, in which one of the coordinates $\phi$ is regarded as that of the motor and another $\chi$ is regarded as a running propeller. The coordinate $\phi$ is defined as a dihedral angle of the $\mathrm{O}^{1}-\mathrm{C}^{2}-\mathrm{C}^{3}-\mathrm{R}^{4}$ group and $\chi$ is specified by a dihedral angle of the $\mathrm{F}^{7}-\mathrm{C}^{6}-$ $\mathrm{C}^{5}-\mathrm{C}^{3}$ group as shown in Fig. 13. The $z$-axis is defined to be along the $\mathrm{C}^{3}-\mathrm{C}^{2}$ bond. The $x$-axis is defined to be on the $\mathrm{C}^{2}-\mathrm{C}^{3}-\mathrm{R}^{4}$ plane. The cyclopentadiene group, which is the main body of the machine, was assumed to be fixed on a surface to reduce the role of entire molecular rotations. In the actual simulation, an alkyl group, $-\mathrm{R}^{4}$, is replaced by $-\mathrm{H}$ for simplicity.

\subsection{Results of quantum dynamics simulation}

The two-dimensional potential energy surface of the molecular machine in the ground state, $V(\phi, \chi)$, was calculated with B3LYP / 6-31+G** (Becke, 1993) in the Gaussian 03 package of programs. All of the other structural parameters were optimized at every two dihedral angles. Three components of the dipole moment function, $\mu_{x}(\phi, \chi), \mu_{y}(\phi, \chi)$ and $\mu_{z}(\phi, \chi)$, were calculated in the same way as that used for calculation of $V(\phi, \chi)$. Quantum chemical calculation shows strong $\phi$ dependence in $\mu_{x}(\phi, \chi)$ and $\mu_{y}(\phi, \chi)$, while $\chi$ dependence is fairly small. This indicates that the motion of $\phi$ is optically active but that of $\chi$ is not. The $z$ component $\mu_{z}(\phi, \chi)$ was nearly constant so that the interaction term is negligible. Thus, 
$\mu(\phi, \chi)$ can be expressed in the same analytical form as Eq. (4) with an amplitude $\mu=2$ Debye. Moments of inertia were assumed to be constant at the most stable molecular structure, $I_{\phi}=2.8 \times 10^{-46} \mathrm{~kg} \mathrm{~m}^{2}$ and $I_{\chi}=1.5 \times 10^{-45} \mathrm{~kg} \mathrm{~m}^{2} . I_{\chi}$ is about five-times heavier than $I_{\phi}$. Figure 14 shows the results of quantum dynamical calculations of the light-driven molecular machine at a low temperature limit. Figure 14a shows the electric field of the pulse which is given as $\mathbf{E}(t)=f(t) \cos (\omega t) \mathbf{e}_{y}$ with envelope function $f(t)$ given by Eq. (11). Here, $\boldsymbol{e}_{y}$ is the unit vector along the $y$-axis as is defined in Fig. 13; frequency $\omega=45 \mathrm{~cm}^{-1}$ was taken as a central frequency of a pulse; $E_{0}=3.7 \mathrm{GVm}^{-1}$ was taken as the amplitude of the envelope function $f(t)$ and $t_{p}=30$ ps was taken as pulse length.

Figure $14 \mathrm{~b}$ shows the instantaneous angular momenta, $L_{\phi}(t)=\operatorname{Tr}\left[\hat{\ell}_{\phi} \hat{\rho}(t)\right]$ (in red) and $L_{\chi}(t)=\operatorname{Tr}\left[\hat{\ell}_{\chi} \hat{\rho}(t)\right]$ (in blue), of the motor and propeller of the machine, respectively. We also defined "expectation values of rotational angles $\phi$ and $\chi$ ", $\phi(t)$ and $\chi(t)$, as indexes of the rotations,

$$
\phi(t)=\frac{1}{I_{\phi}} \int_{0}^{t} d t^{\prime} L_{\phi}\left(t^{\prime}\right)
$$

and

$$
\chi(t)=\frac{1}{I_{\chi}} \int_{0}^{t} d t^{\prime} L_{\chi}\left(t^{\prime}\right) .
$$

They are shown in Fig. 14c in red and blue, respectively. We can clearly see correlated behaviors between the motor and propeller. We can also see how the rotational power is transmitted from the motor to the propeller. The molecule really acts as a single molecular machine.

The dynamic behaviors shown in Fig. 14 can be divided into three stages: early, transient and steady stages. In the early stage with the time range of $0-13$ ps that ends just before the light pulse peak, the motor is subjected to a forced oscillation with large amplitudes in the torsional mode, which is induced by the light pulse, while the propeller just oscillate around the most stable structure with its small amplitudes. In other words, "idling" operates in this stage. This stage can be described by the one-dimensional model: as is the case with Sec. 2.2, it starts to rotate toward the gentle slope side of the asymmetric potential of the chiral molecule. In the transient stage where a bump is located in $\phi(t)$, the rotational direction of the motor is changed. Then $\chi(t)$ starts to increase, i.e., the propeller start to rotate. The rotational directions of the motor and propeller are opposite. This indicates that the aldehyde group and trifluoromethyl group play the role of a bevel gear at the molecular level, although they are not close to each other so as to have direct interactions as can be seen in macroscopic bevel gears. In the stationary stage after the pulse vanishes, the motor and propeller continue to rotate with a constant motion since there are no dephasing processes included.

Figure $14 \mathrm{~d}$ shows the time-dependent expectation values of the following energies: the potential energy, $V(t)=\operatorname{Tr}[\hat{V} \hat{\rho}(t)]$, the kinetic energies, $T_{\phi}(t)=\operatorname{Tr}\left[\hat{T_{\phi}} \hat{\rho}(t)\right]$ and 
$T_{\chi}(t)=\operatorname{Tr}\left[\hat{T}_{\chi} \hat{\rho}(t)\right]$, and the sum of them, $H(t)=V(t)+T_{\phi}(t)+T_{\chi}(t)$. In the early stage, only the wave packet in the direction of $\phi$ is forced to oscillate by the pulse. This can be seen from Fig. 14d, in which both $V(t)$ and $T_{\phi}(t)$ begin to oscillate in a correlative way, while $T_{\chi}(t)$ does not change. In the next stage in which the motion of $\phi$ changes its direction, $T_{\chi}(t)$ begins to increase gradually. This is another proof that the motor and propeller are correlated and that the motion of propeller is induced not by laser pulse but by intramolecular interactions, i.e., non-linear interactions between two torsional modes, $\phi$ and $\chi$.

Temperature effects on the dynamics of the molecular machine were also investigated (Yamaki et al., 2009).

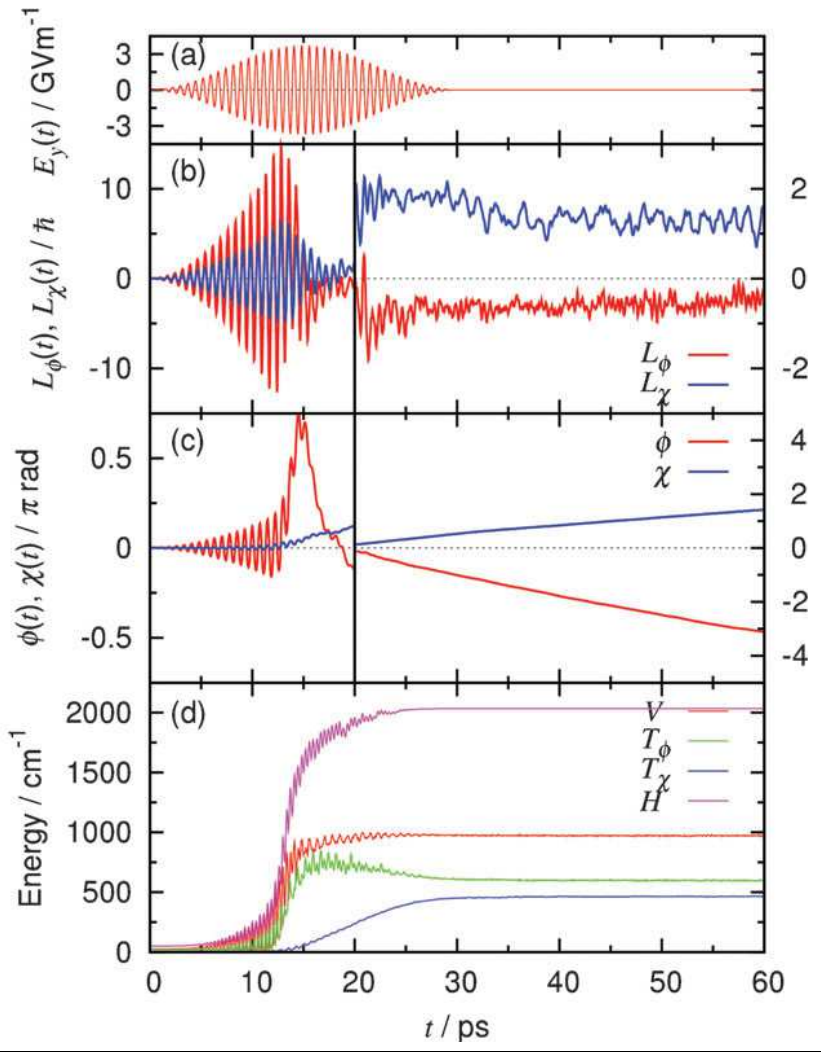

Fig. 14. (a) The y-component of the electric field of the pulse $E_{y}(t)$ used. (b) Quantum mechanical expectation values of angular momentum at $T=0 \mathrm{~K}$ : that of the motor $L_{\phi}(t)$ (in red) and that of the propeller $L_{\chi}(t)$ (in blue). The scale of the vertical axis for $t \leq 20 \mathrm{ps}$ is stretched compared with that for $t \geq 20$ ps. (c) Rotational angle of the motor $\phi(t)$ (in red) and that of the propeller $\chi(t)$ (in blue). (d) Quantum mechanical expectation values of energies: potential energy $V(t)$ (in red), kinetic energy of $\mathrm{f}$ rotation, $T_{\phi}(t)$ (in green), and of $\chi$, $T_{\chi}(t)$ (in blue), and the sum of them (in magenta). Reproduced with permission from Phys. Chem. Chem. Phys., 11, 1662 (2009). 
Finally, we briefly discuss the mechanism of formation of the bevel gear in the molecular machine. Quantum dynamics simulation shows that the rotational wave packet of the motor, which is created by a laser pulse, is transferred to that of the propeller. Such a correlated behavior can be quantum mechanically explained in terms of a rotational coherence transfer mechanism. We note that the correlated groups, the motor and propeller, are located at a distance of $2.3 \AA$. This is long compared with distance of $1.4 \AA(1.5 \AA)$ between carbon atoms of a double (single) bond. There may be two possible mechanisms: one originates from through-conjugation and the other from through-space interactions. It should be noted that the conjugation of the machine is restricted to its main body. Therefore, the through-space interaction mechanism is the most likely mechanism. Further detailed analysis is needed to confirm the transfer mechanism.

\section{Summary and perspectives}

Results of theoretical treatments on quantum dynamics and quantum control of laser-driven chiral molecular motors were presented. First, fundamental principles for unidirectional motions of chiral molecular motors driven by linearly polarized (nonhelical) laser pulses were described. Similarities and differences between the mechanism for driving directional motions in the case of Brownian motors for bio-motors and in the case of chiral molecular motors developed in our study were clarified. In bio-motors, the unidirectional motions are explained in terms of so-called nonequilibrium fluctuations of a Brownian motion with a saw-toothed ratchet potential, while chiral molecular motors, which are characterized by asymmetric potential similar to a saw-toothed ratchet potential, are driven in a unidirectional way by time-dependent periodic perturbations of linear polarized lasers with no angular momentum. Here, the magnitudes of the perturbations are large compared with those of interactions between molecular motors and heat bath modes, which makes the system different. Quantum dynamics simulations showed that the directional motion is determined by molecular chirality. This supports the mechanism for unidiredtional motions of chiral motors. We call the direction of the gentle slope of the asymmetric potential the intuitive direction for the unidirectional motion.

Secondly, after reviewing a quantum control theory for driving a molecular rotor with a designated unidirectional motion, we presented the results of quantum control of chiral molecular rotors. Pulse shapes for driving rotational motions in the intuitive direction or the counter-intuitive direction were found with the help of the quantum control theory. The mechanisms of the intuitive and counter-intuitive rotations were clarified by analyzing nuclear wave packet motions. We restricted ourselves to simple real molecules rather than complicated molecular systems to elucidate features of quantum control of molecular motors. We also presented an effective method for controlling unidirectional motions via an electronic excited state of chiral motors.

Thirdly, results of theoretical design of the smallest laser-driven molecular machine were presented. The smallest chiral molecular machine has an optically driven motor and a running propeller on its body. The mechanism of the transmission of driving forces from the motor to the propeller was clarified by using a quantum dynamical treatment.

In this chapter, the quantum control procedures were applied to small molecular motors with the rotary part consisting of a simple, optically active group connected to the body by a single bond. Molecular machines with nano-scale dimension have now been synthesized and wait for their operation by external forces. One of the next subjects is to demonstrate 
that these artificial machines can be driven by laser pulses. For example, laser pulses designed by quantum control procedures will be able to control their motions: acceleration or slowdown, forward or reverse motions and even turning directions. In principle, laser light can control coherent directed motions of assembled molecules as well. This can realize coherent collective precession of molecular rotors with chiral propellers (Kinbara \& Aida, 2005; Tabe \& Yokoyama, 2003). Similarly, it would be interesting to control a molecular motor in a cage, which is a model of molecular gyroscope (Bedard \& Moore, 1995; Dominguez et al., 2002; Setaka et al., 2007). Another interesting subject is to apply control procedures described in this chapter to bio-systems with a micrometer dimension. For example, results of laser-induced rotational motions of both normal and malaria-infected red blood cells in various medium solutions have recently been reported (Bambardekar et al., 2010). The experiments were carried out by using linearly polarized laser pulses. It was found that the shape anisotropy of red blood cells induces rotations in optically trapped red blood cells. The rotational dynamics depends on the shape changes, which are realized by altering the experimental conditions such as osmolarity of the medium containing the cells. Differences in rotational motions between normal and malaria-infected red blood cells have been identified as well. Such a complicated rotational dynamics can be analyzed by using laser optimal control procedures, which can be used as a fast diagnostic method for malariainfected red blood cells.

\section{References}

Abrahams, J. P.; Leslie, A. G. W.; Lutter, R. \& Walker, J. E. (1994). Structure at $2.8 \AA$ resolution of $\mathrm{F}_{1}$-ATPase from bovine heart mitochondria, Nature, 370, (Aug 25 1994) 621-628, 0028-0836

Assion, A.; Baumert, T.; Bergt, M.; Brixner, T.; Kiefer, B.; Seyfried, V.; Strehle, M. \& Gerber, G. (1998). Control of chemical reactions by feedback-optimized phase-shaped femtosecond laser pulses, Science, 282, 5390, (Oct 30 1998) 919-922, 0036-8075

Astumian, R. D. \& Häanggi, P. (2002). Brownian motors, Physics Today, 55, 11, (Nov 2002) 3339, 0031-9228

Balzani, V.; Credi, A. \& Venturi, M. (2008). Molecular machines working on surfaces and at int erfaces, Chem. Phys. Chem. , 9, 2, (Feb 1 2008) 202-220, 1439-4235

Bambardekar, K.; Dharmadhikari, J.; Dharmadhikari, A. K.; Yamada, T.; Kato, T.; Kono, H.; Fujimura, Y.; Sharma, S. \& Mathur, D., (2010). Shape anisotropy induces rotations in optically trapped red blood cells, J. Biomed. Opt., 15. 4, (Jul/Aug, 2010)041504, 1083-3668

Bandrauk, A. D.; Fujimura, Y. \& Gordon, R. J. (2002). ACS Symposium Series 821, Laser Control and Manipulation of Molecules, American Chemical Society, 0-8412-3786-7, Washington, DC

Becke, A. D. (1993). A new mixing of Hartree--Fock and local density--functional theories, J. Chem. Phys., 98, 2, (1993) 1372--1377, 0021-9606

Bedard, T. C. \& Moore, J. S. (1995). Design and synthesis of a molecular turnstile, J.Am. Chem. Soc., 117, 43 (1995) 10662-10671, 0002-7863

Boyer, P. D. (1993). The binding change mechanism for ATP synthase - Some probabilities and possibilities, Biochimica et Biophysica Acta, 1140, 3, (Jan 8 1993) 215-250, 0005-2728

Choi, S. E. \& Light, J. C. (1989). Use of the discrete variable representation in the quantum dynamics by a wave packet propagation: Predissociation of $\mathrm{NaI}\left({ }^{1} \Sigma_{0}+\right) \rightarrow \mathrm{NaI}\left(0^{+}\right)$ $\rightarrow \mathrm{Na}\left({ }^{2} \mathrm{~S}\right)+\mathrm{I}\left({ }^{2} \mathrm{P}\right)$, J. Chem. Phys. 90, 5, (Mar 1 1989) 2593-2599, 0021-9606 
Dominguez, Z.; Dang, H.; Strouse, M. J. \& Garcia-Garibay, M. A., (2002). Molecular "Compasses" and "Gyroscopes". I. Expedient synthesis and solid state dynamics of an open rotor with a bis(triarylmethyl) frame J. Am. Chem. Soc., 124, 11, (Mar 20 2002) 2398-2399, 0002-7863

Feringa, B. L.; van Delden, R. A.; Koumura, N. \& Geertsema, E. M. (2000). Chiroptical molecular switches, Chem. Rev., 100, 5, (May 2000) 1789-1816, 0009-2665

Frisch, M. J.; Trucks, G. W.; Schlegel, H. B.; Scuseria, G. E.; Robb, M. A.; Cheeseman, J. R.; Zakrzewski, V. G.; Montgomery, J. A.; Stratmann, R. E.; Burant, J. C.; Dapprich, S.; Millam, J. M.; Daniels, A. D.; Kudin; K. N.; Strain, M. C.; Farkas, O.; Tomasi, J.; Barone, V.; Cossi, M.; Cammi, R.; Mennucci, B.; Pomelli, C.; Adamo, C.; Clifford, S.; Ochterski, J.; Petersson, G. A.; Ayala, P. Y.; Cui, Q.; Morokuma, K.; Malick, D. K.; Rabuck, A. D.; Raghavachari, K.; Foresman, J. B.; Cioslowski, J.; Ortiz, J. V.; Stefanov, B. B.; Liu, G.; Liashenko, A.; Piskorz, P.; Komaromi, I.; Gomperts, R.; Martin, R. L.; Fox, D. J.; Keith, T.; Al-Laham, M. A.; Peng, C. Y.; Nanayakkara, A.; Gonzalez, C.; Challacombe, M.; Gill, P. M. W.; Johnson, B. G.; Chen, W.; Wong, M. W.; Andres, J. L.; Head-Gordon, M.; Replogle, E. S. \& Pople, J. A. (1998). Gaussian98 (RevisionA.7), Gaussian Inc., Pittsburgh, PA

Fujimura, Y.; González, L..; Kröner, D.; Manz, J.; Mehdaoui, I. \& Schmidt, B. (2004). Quantum ignition of intramolecular rotation by means of IR+UV laser pulses, Chem. Phys. Lett., 386, 4-6, (Mar 11 2004) 248-253, 0009-2614

Gordon, R. A. \& Fujimura, Y. (2002). Coherent control of chemical reactions, In: Encyclopedia of Physical Science and Technology, Robert A. Meyers (Ed.), 207--231, Academic Press, 978-0-12-227410-7, San Diego

Gouliemakis, E.; Uiberacker, M.; Kienberger, R.; Baltuska, A.; Yakovlev, V.; Scrinzi, A.; Westerwalbesloh, T.; Kleineberg, U.; Heinzmann, U.; Drescher, M.; \& Krausz, F., (2004). Direct measurement of light waves, Science, 305, (Aug 27 2004) 1267-1269, 0036-8075

Hoki, K.; Ohtsuki, Y. \& Fujimura, Y. (2001). Locally designed pulse shaping for selective preparation of enantiomers from their racemate, J. Chem. Phys., 114, 4,(Jan 22 2001) 1575-1581, 0021-9606

Hoki, K.; Yamaki, M.; Koseki, S. \& Fujimura, Y. (2003) Molecular motors driven by laser pulses: Role of molecular chirality and photon helicity, J. Chem. Phys., 118, 2, (Jan 8 2003) 497-504, 0021-9606

Hoki, K.; Yamaki, M. \& Fujimura, Y. (2003). Chiral molecular motors driven by a nonhelical laser pulse, Angew. Chem. Int. Ed., 42, 26, (Jul 7 2003) 2975-2978, 1433-7851

Hoki, K.; Yamaki, M.; Koseki, S. \& Fujimura, Y. (2003). Mechanism of unidirectional motions of chiral molecular motors driven by linearly polarized pulses, J. Chem. Phys., 119, 23, (Dec 15 2003) 12393-12398, 0021-9606

Hoki, K.; Sato, M.; Yamaki, M.; Sahnoun, R; González, L.; Koseki, S. \& Fujimura, Y. (2004). Chiral molecular motors ignited by femtosecond pump-dump laser pulses, J. Phys. Chem. B, 108, 15, (Apr 15 2004) 4916-4921, 1520-6106

Jülicher, F.; Ajdari, A. \& Prost, J. (1997). Modeling molecular motors, Rev. Mod. Phys., 69, 4, (Oct 1997) 1269-1281, 0034-6861

Kay, E. R.; Leigh, D. A. \& Zerbetto, F. (2007). Synthetic molecular motors and mechanical machines, Angew. Chem. Int. Ed., 46, 1-2, (2007) 72-191, 1433-7851

Kinbara, K. \& Aida, T. (2005). Toward intelligent molecular machines: Directed motions of biological and artificial molecules and assemblies, Chem. Rev., 105, 4, (Apr 2005) 1377-1400, 0009-2665 
Kosloff, R.; Rice, S. A.; Gaspard, P.; Tersigni, S. \& Tannor, D. J. (1989). Wavepacket dancing Achieving chemical selectivity by shaping light-pulses, Chem. Phys., 139, 1, (Dec 1 1989) 201-220, 0301-0104

Lindblad, G. (1976). Generators of quantum dynamical semigroups, Commun. Math. Phys., 48, 2, (1976) 119--130, 0010-3616

Ohtsuki, Y.; Zhu, W. \& Rabitz, H. (1999). Monotonically convergent algorithm for quantum optimal control with dissipation, J. Chem. Phys., 110, 20, (May 22 1999) 9825-9832, 0021-9606

Potter, E. D.; Herek, J. L.; Pedersen, S.; Liu, Q. \& Zewail, A. H. (1992). Femtosecond laser control of a chemical-reaction, Nature, 355, 6355, (Jan -2 1992) 66-68, 0028-0836

Reimann, P. (2002). Brownian motors: noisy transport far from equilibrium, Physics Reports, 361, 2-4, (Apr 2002) 57-265, 0370-1573

Rice, S. A. \& Zhao, M. (2000). Optimal Control of Molecular Dynamics, John Wiley \& Sons Inc, 978-0-471-35423-9, New York

Roncaglia, R. \& Tsironis, G. P. (1998). Discrete quantum motors, Phys. Rev. Lett., 81, 1, (Jul 6 1998) 10-13, 0031-9007

Ross, B. O. (1987). The complete active space self-consistent field method and its applications in electronic structure calculations, In: Ab initio methods in quantum chemistry-II, volume 69 of advances in chemical physics, Lawly, K. P. (ed.) 339-445, Wily, 0065-2385, New York

Setaka, W.; Ohmizu, S.; Kabuto, C. \& Kira, M. (2007). A molecular gyroscope having phenylene rotator encased in three-spoke silicon-based stator, Chem. Lett., 36, 8, (Aug 5 2007) 1076-1077, 0366-7022

Shapiro, M. \& Brumer, P. (2000). Coherent control of atomic molecular, and electronic processes, In: Advances in atomic molecular and optical physics Vol.42, (2000), Ed. Bederson, B. \& Walther, H., 287-345, Academic Press Inc., 9780120038428, San Diego

Shi, S. H. \& Rabitz, H. (1990). Quantum-mechanical optimal-control of physical observables in microsystems, J. Chem. Phys., 92 , 1, (Jan 1 1990) 364-376, 0021-9606

Sugawara, M. \& Fujimura, Y. (1994). Control of quantum dynamics by a locally optimized laser field - application to ring puckering isomerization, J. Chem. Phys., 100, 8, (Apr 15 1994), 5646-5655, 0021-9606

Tabe, Y.; Yokoyama, H. (2003). Coherent collective precession of molecular rotors with chiral propellers, Nature Materials, 2, 12, (Dec 2003) 806-809, 1476-1122

Yamaki, M; Hoki, K.; Ohtsuki, Y.; Kono, H. \& Fujimura, Y. (2005). Quantum control of a chiral molecular motor driven by laser pulses, J. Am. Chem. Soc., 127, 20, (May 25 2005) 7300-7301, 0002-7863

Yamaki, M; Hoki, K.; Ohtsuki, Y.; Kono, H. \& Fujimura, Y. (2005). Quantum control of a unidirectional rotation of a chiral molecular motor, Phys. Chem. Chem. Phys. 7, 9, (May 7 2005) 1900-1904, 1463-9076

Yamaki, M; Hoki, K.; Kono, H. \& Fujimura, Y. (2008). Quantum control of a chiral molecular motor driven by femtosecond laser pulses: Mechanisms of regular and reverse rotations, Chem. Phys., 347, 1-3, (May 23 2008) 272-278, 0301-0104

Yamaki, M; Nakayama, S.; Hoki, K.; Kono, H. \& Fujimura, Y. (2009). Quantum dynamics of light-driven chiral molecular motors, Phys. Chem. Chem. Phys. 11, 11, (2009) 16621678, 1463-9076

Zewail, A. \& Bernstein, R. (1992). Real-Time Laser femtochemistry viewing the transition from reagents to products, In: The Chemical Bond Structure and Dynamics, Zawail, A., (Ed.) 223-279, Academic Press, Inc., 0-12-779620-7, San Diego, CA 


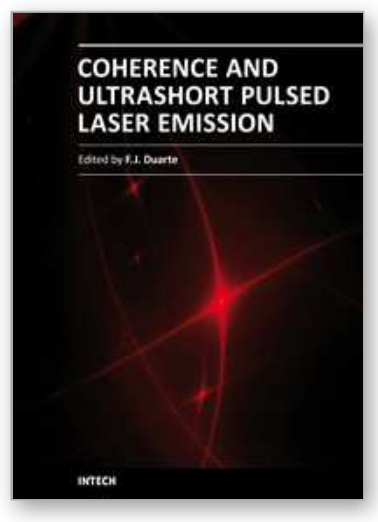

\section{Coherence and Ultrashort Pulse Laser Emission}

Edited by Dr. F. J. Duarte

ISBN 978-953-307-242-5

Hard cover, 688 pages

Publisher InTech

Published online 30, November, 2010

Published in print edition November, 2010

In this volume, recent contributions on coherence provide a useful perspective on the diversity of various coherent sources of emission and coherent related phenomena of current interest. These papers provide a preamble for a larger collection of contributions on ultrashort pulse laser generation and ultrashort pulse laser phenomena. Papers on ultrashort pulse phenomena include works on few cycle pulses, high-power generation, propagation in various media, to various applications of current interest. Undoubtedly, Coherence and Ultrashort Pulse Emission offers a rich and practical perspective on this rapidly evolving field.

\section{How to reference}

In order to correctly reference this scholarly work, feel free to copy and paste the following:

Masahiro Yamaki, Kunihito Hoki, Yuichi Fujimura and Sheng H. Lin (2010). Quantum Control of Laser-driven Chiral Molecular Motors, Coherence and Ultrashort Pulse Laser Emission, Dr. F. J. Duarte (Ed.), ISBN: 978953-307-242-5, InTech, Available from: http://www.intechopen.com/books/coherence-and-ultrashort-pulselaser-emission/quantum-control-of-laser-driven-chiral-molecular-motors

\section{INTECH}

open science | open minds

\section{InTech Europe}

University Campus STeP Ri

Slavka Krautzeka 83/A

51000 Rijeka, Croatia

Phone: +385 (51) 770447

Fax: +385 (51) 686166

www.intechopen.com

\section{InTech China}

Unit 405, Office Block, Hotel Equatorial Shanghai

No.65, Yan An Road (West), Shanghai, 200040, China

中国上海市延安西路65号上海国际贵都大饭店办公楼 405 单元

Phone: +86-21-62489820

Fax: +86-21-62489821 
(C) 2010 The Author(s). Licensee IntechOpen. This chapter is distributed under the terms of the Creative Commons Attribution-NonCommercialShareAlike-3.0 License, which permits use, distribution and reproduction for non-commercial purposes, provided the original is properly cited and derivative works building on this content are distributed under the same license. 